\title{
Dirac spectrum and chiral condensate for QCD at fixed $\theta$ angle
}

\author{
M. Kieburg, ${ }^{1, *}$ J. J. M. Verbaarschot, ${ }^{2, \dagger}$ and T. Wettig ${ }^{3,+}$ \\ ${ }^{1}$ Department of Physics, University of Bielefeld, 33501 Bielefeld, Germany \\ ${ }^{2}$ Department of Physics and Astronomy, The State University of New York (SUNY), \\ Stony Brook, New York 11794, USA \\ ${ }^{3}$ Department of Physics, University of Regensburg, 93040 Regensburg, Germany
}

(Received 18 January 2019; published 30 April 2019)

\begin{abstract}
We analyze the mass dependence of the chiral condensate for QCD at nonzero $\theta$ angle and find that in general the discontinuity of the chiral condensate is not on the support of the Dirac spectrum. To understand this behavior we decompose the spectral density and the chiral condensate into contributions from the zero modes, the quenched part, and a remainder which is sensitive to the fermion determinant and is referred to as the dynamical part. We obtain general formulas for the contributions of the zero modes. Expressions for the quenched part, valid for an arbitrary number of flavors, and for the dynamical part, valid for one and two flavors, are derived in the microscopic domain of QCD. We find that at nonzero $\theta$ angle the quenched and dynamical parts of the Dirac spectral density are strongly oscillating with an amplitude that increases exponentially with the volume $V$ and a period of order of $1 / V$. The quenched part of the chiral condensate becomes exponentially large at $\theta \neq 0$, but this divergence is canceled by the contribution from the zero modes. The oscillatory behavior of the dynamical part of the density is essential for moving the discontinuity of the chiral condensate away from the support of the Dirac spectrum. As important by-products of this work we obtain analytical expressions for the microscopic spectral density of the Dirac operator at nonzero $\theta$ angle for both one- and two-flavor QCD with nonzero quark masses.
\end{abstract}

DOI: $10.1103 /$ PhysRevD.99.074515

\section{INTRODUCTION}

Topology in the form of instantons and dyons is an important ingredient of the QCD vacuum [1-3]. The main reason is that the Dirac operator for field configurations with nonzero topological charge has a generic number of exact zero modes, which induce the chiral condensate for massless quarks. At nonzero quark mass, the total number of instantons and anti-instantons is even more important. This number scales with the four-dimensional spacetime volume $V$, unlike the topological charge, which scales as $\sqrt{V}$. If instantons and anti-instantons are not strongly overlapping, they give rise to near-zero modes [4] which determine the value of the chiral condensate. Given the importance of topology for the QCD partition function, it is puzzling that the conjugate parameter, the so-called $\theta$ angle, is consistent with zero according to all available

\footnotetext{
*mkieburg@physik.uni-bielefeld.de

jacobus.verbaarschot@stonybrook.edu

*tilo.wettig@ur.de
}

Published by the American Physical Society under the terms of the Creative Commons Attribution 4.0 International license. Further distribution of this work must maintain attribution to the author(s) and the published article's title, journal citation, and DOI. Funded by SCOAP. experimental evidence. Nevertheless, theories with nonzero $\theta$ angle have received a great deal of attention both as theories beyond the standard model as well as from a purely theoretical perspective [5-10].

In [11,12] we have resolved an interesting apparent puzzle for one-flavor QCD at zero $\theta$ angle: the chiral condensate should be independent of the sign of the quark mass $m$, but the condensate expressed in terms of the Dirac eigenvalues appears to be an odd function of $m$. The point is that this function still needs to be averaged over gauge fields, and this average depends on the quark mass through the fermion determinant in such a way that the chiral condensate eventually becomes an even function of $m$. This resolution is reviewed in Sec. II: at negative quark mass, the statistical weight in the average over gauge fields becomes negative, which leads to exponentially large oscillations that can move the discontinuity of the chiral condensate away from the support of the Dirac spectrum and could be shown to yield a mass-independent condensate. In the present paper we extend the work of [11,12] to arbitrary $\theta$ angle and to more than one flavor, as already sketched in [13]. Let us emphasize that for nonzero $\theta$ the statistical weight is not only negative but becomes complex.

To be able to obtain explicit results, most of our calculations are performed in the $\varepsilon$ domain of QCD (also called the microscopic domain). In this domain the Compton 
wavelength of the pion is much larger than the size of the box so that the kinetic term of the chiral Lagrangian can be ignored and only the mass term remains. This chiral Lagrangian can also be obtained from a random matrix theory with the same global symmetries as QCD [14,15], which makes it possible to find analytical expressions for the spectral density of the Dirac operator using powerful random matrix techniques. Another benefit of working in the $\varepsilon$ domain is that the chiral condensate can be obtained from the spectral density without any additional regularization. This is important because the spectral density of the Dirac operator is renormalization-group and gauge invariant, while the chiral condensate is only gauge invariant.

The structure of this paper is as follows. In Sec. II we review the mass dependence of the chiral condensate for one- and two-flavor QCD. The sign problem for QCD at nonzero $\theta$ angle is discussed in Sec. III. A decomposition of the spectral density and the chiral condensate is introduced in Sec. IV. In Sec. V we derive general analytical expressions for the contributions of the zero modes and of the quenched part of the Dirac spectrum to the chiral condensate and show that each of them increases exponentially with the volume at nonzero $\theta$ angle, but that their sum remains finite. The one-flavor case is worked out in detail in Sec. VI, where we also obtain an expression for the spectral density of the Dirac operator at fixed $\theta$ angle. The two-flavor case is discussed in Sec. VII, where we derive analytical expressions for the spectral density and the chiral condensate at fixed $\theta$ angle. Concluding remarks are made in Sec. VIII, and technical details are given in several Appendixes. In particular, in Appendix A we obtain identities for sums of products of three and four Bessel functions which, as far as we know, are new.

Some notes on notation: on the macroscopic scale, the Dirac eigenvalues and the quark masses are denoted by $\lambda$ and $m$, while on the microscopic scale they are denoted by $x$ and $u$, respectively. For functions of these variables, such as the partition function $Z$, the spectral density $\rho$ or the chiral condensate $\Sigma$, we use the same symbol on the macroscopic and the microscopic scales to simplify the notation. The corresponding functions are of course different, but it should be clear from the arguments of the function what is meant in every case. Also, when we give results for partition functions, we drop irrelevant normalization factors.

\section{REVIEW OF KNOWN RESULTS}

We consider QCD with $N_{f}$ quark flavors and quark mass matrix $M=\operatorname{diag}\left(m_{1}, \ldots, m_{N_{f}}\right)$, which we allow to be complex for the time being. The mass matrix appears in the QCD Lagrangian in the form $\bar{\psi}_{R} M \psi_{L}+\bar{\psi}_{L} M^{\dagger} \psi_{R}$, where $\psi_{R / L}$ denotes quark fields of definite chirality. For a given gauge-field configuration with topological charge $\nu$, the fermion determinant is

$$
\prod_{\lambda_{n}>0} \operatorname{det}\left(\lambda_{n}^{2}+M M^{\dagger}\right) \times \begin{cases}\operatorname{det}^{\nu} M, & \nu \geq 0, \\ \operatorname{det}^{-\nu} M^{\dagger}, & \nu<0,\end{cases}
$$

where the $\lambda_{n}$ are the eigenvalues of the Dirac operator, and the second factor is due to the presence of $|\nu|$ exact zero modes. The partition function for fixed topological charge $\nu$ is given by the average of the fermion determinant over gauge-field configurations with fixed $\nu$. Defining

$$
\hat{Z}_{|\nu|}(|M|)=\left\langle\prod_{\lambda_{n}>0} \operatorname{det}\left(\lambda_{n}^{2}+M M^{\dagger}\right)\right\rangle_{\nu},
$$

which only depends on $|M|=\operatorname{diag}\left(\left|m_{1}\right|, \ldots,\left|m_{N_{f}}\right|\right)$ and $|\nu|$, the partition function reads

$$
Z_{\nu}(M)=\hat{Z}_{|\nu|}(|M|) \times \begin{cases}\operatorname{det}^{\nu} M, & \nu \geq 0, \\ \operatorname{det}^{-\nu} M^{\dagger}, & \nu<0 .\end{cases}
$$

The partition function at fixed $\theta$ angle is then given by

$$
\begin{aligned}
Z(M, \theta) & =\sum_{\nu=-\infty}^{\infty} e^{i \nu \theta} Z_{\nu}(M) \\
& =\sum_{\nu=-\infty}^{\infty} e^{i \nu\left(\theta+\sum_{k=1}^{N_{f}} \varphi_{k}\right)} \operatorname{det}|M|^{|\nu|} \hat{Z}_{|\nu|}(|M|),
\end{aligned}
$$

where $m_{k}=\left|m_{k}\right| e^{i \varphi_{k}}$ defines the phase $\varphi_{k}$ of $m_{k}$. It is clear that $Z(M, \theta)$ is a periodic function of $\theta$, and if $\sum_{k} \varphi_{k}$ is a multiple of $\pi$, it is also even in $\theta$. It only depends on the sum of the phases of the quark masses, and this sum can be absorbed in a redefinition of the $\theta$ angle. The same statement is true for the spectral density, whose mass dependence comes only from the fermion determinant in the statistical measure.

Therefore, from a mathematical point of view, it suffices to derive results for real and non-negative quark masses. However, we will sometimes consider the "physical" situation where one of the quark masses is taken to be negative. This case can be obtained by shifting $\theta \rightarrow \theta+\pi$ in the mathematical result. Nevertheless, our results assume $m_{k} \geq 0$ unless stated otherwise.

The chiral condensate of flavor $k$ is defined as

$$
\begin{aligned}
\Sigma\left(m_{k}\right) & =-\left\langle\bar{\psi}_{k} \psi_{k}\right\rangle=-\left\langle\bar{\psi}_{k R} \psi_{k L}\right\rangle-\left\langle\bar{\psi}_{k L} \psi_{k R}\right\rangle \\
& =\frac{1}{N_{d} V}\left(\frac{d}{d m_{k}}+\frac{d}{d m_{k}^{*}}\right) \log Z(M),
\end{aligned}
$$

where we have suppressed the dependence of $\Sigma\left(m_{k}\right)$ on $M$ and where $N_{d}$ (with $d$ for "degenerate") is the number of quarks whose mass equals $m_{k}$. Note that Eq. (5) is valid both at fixed $\nu$ and at fixed $\theta$. Let us make two remarks here. First, for a real and negative mass, the derivatives in Eq. (5) simply lead to an extra sign (compared with the result for a positive mass). Second, for a genuinely complex 
mass $m_{k}=\left|m_{k}\right| e^{i \varphi_{k}}$, one can rewrite the derivatives in terms of $\left|m_{k}\right|$ and $\varphi_{k}$. For the partition function (4) at fixed $\theta$ the derivative with respect to $\varphi_{k}$ can be rewritten as a derivative with respect to $\theta$.

Let us begin with the case of one flavor of mass $m$. Since the free energy is extensive in $V$, the QCD partition function, obtained by expanding the action to lowest order in $m$, is given by [5]

$Z(m, \theta)=Z(m=0, \theta) \exp \left[m V \Sigma \cos \theta+O\left(m^{2} V\right)\right]$,

where $\Sigma$ is the absolute value of the chiral condensate in the limit $m=0$ and $\theta=0$. Since the mass-independent factor $Z(m=0, \theta)$ does not contribute to the chiral condensate we will ignore it below. Equation (6) is valid for both positive and negative quark masses. This has the consequence that the chiral condensate from Eq. (5),

$$
\Sigma(m)=\Sigma \cos \theta
$$

does not change sign when $m$ becomes negative. Assuming nonvanishing $\Sigma$, at first sight this appears to be in contradiction with the Banks-Casher formula [16], which expresses the chiral condensate in terms of the eigenvalues $\lambda_{n}$ of the Dirac operator starting from the relation

$$
\Sigma(m)=\left\langle\frac{1}{V} \sum_{n} \frac{1}{i \lambda_{n}+m}\right\rangle,
$$

where the average is over gauge-field configurations. Equation (8) is valid both at fixed $\nu$ and at fixed $\theta$. The eigenvalues either are zero, giving a term proportional to $1 / m$, or occur in pairs $\pm \lambda_{n}$, which yield terms of the form $2 m /\left(\lambda_{n}^{2}+m^{2}\right)$. Thus the function $\Sigma(m)$ appears to be odd in $m$. The resolution of this puzzle is that the statistical weight in Eq. (8) contains the fermion determinant, which leads to an additional mass dependence. This resolution was fully worked out in Refs. [11,12] by an explicit computation of the spectral density and the chiral condensate in the $\varepsilon$ domain of QCD. Employing identities for sums of products of Bessel functions, the expressions could be summed to give the spectral density at $\theta=0$. For negative mass the resulting expression is increasing exponentially with $V$ and oscillating with a period that scales as $1 / V$. As we know from QCD at nonzero chemical potential [17], exactly such behavior of the spectral density can eliminate a discontinuity of the chiral condensate. However, as has already been observed in [18], the contributions of both the zero modes and the nonzero modes diverge exponentially with the volume. It turns out $[11,12]$ that these divergent contributions cancel identically, resulting in a chiral condensate that remains constant in the $\varepsilon$ domain, i.e., for $m \Lambda_{\mathrm{QCD}} \sqrt{V} \ll 1$.

Let us turn to two-flavor QCD. In this case the full flavor symmetry is $\mathrm{U}(2) \times \mathrm{U}(2)$, with the axial $\mathrm{U}(1)$ group broken by the anomaly and the $\mathrm{SU}(2) \times \mathrm{SU}(2)$ subgroup broken spontaneously by the chiral condensate. In the $\varepsilon$ domain of QCD, the partition function of the resulting Nambu-Goldstone modes, which interact according to a chiral Lagrangian, simplifies to [5]

$$
Z(M, \theta)=\int_{\mathrm{SU}\left(N_{f}\right)} d U \exp \left[V \Sigma \operatorname{Re}\left(e^{i \theta / N_{f}} \operatorname{Tr} M U\right)\right],
$$

which actually holds for any $N_{f}$. The measure $d U$ is the normalized Haar measure. In the thermodynamic limit, the $U$ field aligns itself with the chiral condensate. For $N_{f}=2$, the simplest case is when the two masses are equal to a common mass $m$. For $m \cos (\theta / 2)>0$ the saddle-point solution is $U=\mathbb{1}$, but for $m \cos (\theta / 2)<0$ it is given by $U=-1$. To leading order in the thermodynamic limit this results in

$$
\log Z(m, m, \theta) \stackrel{|m| V \Sigma \gg 1}{\approx} 2 V \Sigma|m \cos (\theta / 2)| .
$$

Because of the absolute value, the chiral condensate as defined in Eq. (5) acquires a discontinuity at $m=0$,

$$
\Sigma(m) \stackrel{|m| V \Sigma \gg 1}{\approx} \operatorname{sign}(m) \Sigma|\cos (\theta / 2)| .
$$

Another simple case is that of unequal quark masses and $\theta=0$, where we have in leading order [5]

$$
\log Z\left(m_{1}, m_{2}, \theta=0\right) \stackrel{\left|m_{k}\right| V \Sigma \gg 1}{\approx} V \Sigma\left|m_{1}+m_{2}\right| .
$$

The phase diagram of this case is shown in Fig. 1. The two phases are separated by the line $m_{1}+m_{2}=0$ on which the pion mass becomes zero, known as the Dashen phenomenon [19]. For three flavors, this line changes to a finite region where pions condense and $C P$ symmetry is spontaneously broken [20]. It can also become a finite region when pion condensation occurs in the case of Wilson fermions [21].

The phase diagram at fixed $\theta$ has to be contrasted to the one at fixed topological charge $\nu$, where we have in leading order [5]

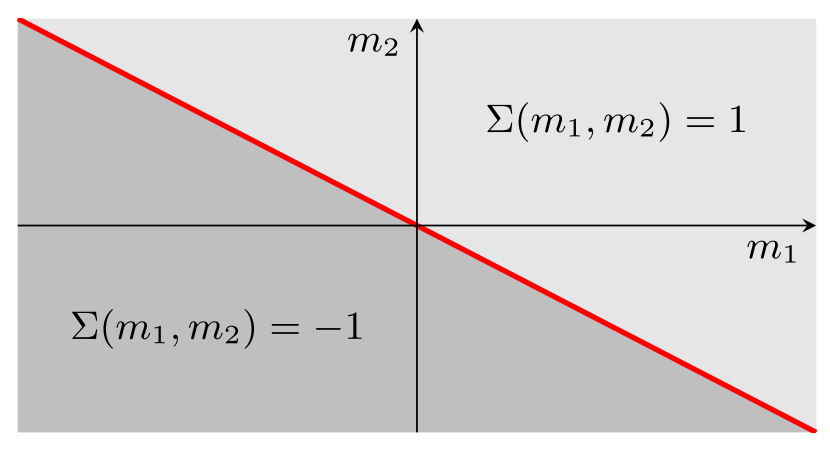

FIG. 1. Phase diagram of the two-flavor theory at $\theta=0$ in the plane of the two quark masses. 

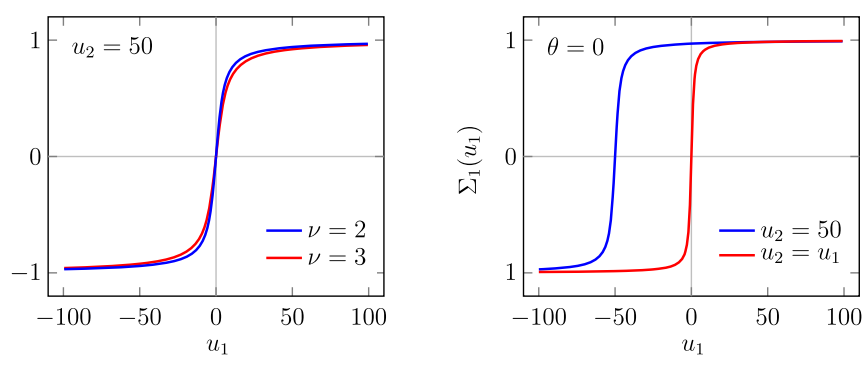

FIG. 2. Mass dependence of the chiral condensate for twoflavor QCD at fixed topological charge $\nu$ (left) and at fixed $\theta=0$ (right). In the thermodynamic limit all curves become discontinuous. Note that we use the dimensionless masses $u_{k}=m_{k} V \Sigma$. The notation $\Sigma_{1}$ indicates that we differentiate with respect to the first quark mass $u_{1}$.

$$
\log Z_{\nu}\left(m_{1}, m_{2}\right) \stackrel{\left|m_{k}\right| V \Sigma \gg 1}{\approx} V \Sigma\left(\left|m_{1}\right|+\left|m_{2}\right|\right)
$$

The sign of the chiral condensate at fixed $\nu$ always changes sign when one of the masses changes its sign, unlike at fixed $\theta=0$, where the sign change occurs at the Dashen line $m_{1}=-m_{2}$. One aim of the present work is to understand this difference. In Fig. 2 we highlight the different behavior of the two-flavor chiral condensate at fixed topological charge $\nu$ (left) and fixed $\theta$ angle (right).

Finally, let us mention one particular limit of the twoflavor case. When one of the quark masses becomes large, the two-flavor theory reduces to the one-flavor theory. In the chiral Lagrangian we then have

$$
U \rightarrow\left(\begin{array}{cc}
e^{-i \varphi} & 0 \\
0 & e^{i \varphi}
\end{array}\right)
$$

with $\varphi$ fixed at $\varphi=\theta / 2$, resulting in the one-flavor partition function (6).

\section{SIGN PROBLEM}

Generically, QCD at nonzero $\theta$ angle has a sign problem, which originates from the weight factor $\exp \left[i \nu\left(\theta+\sum_{k} \varphi_{k}\right)\right]$ in Eq. (4). The sign problem is absent only for $\cos \left(\theta+\sum_{k} \varphi_{k}\right)=1$, and it is most severe for $\cos \left(\theta+\sum_{k} \varphi_{k}\right)=-1$, in which case the weight factor is $(-1)^{\nu}$. For real (but possibly negative) quark masses, these two conditions translate into $\cos \theta= \pm \operatorname{sign} \operatorname{det} M$.

While these two extreme cases are already apparent from Eq. (4), the severity of the sign problem in the general case can be measured by the ratio of the partition function with a phase and the phase-quenched partition function. Explicit analytical results for this ratio can be obtained in the $\varepsilon$ domain.

For one flavor the ratio is given by

$$
\exp [-\Delta F(m, \theta)]=\frac{Z(m, \theta)}{Z(|m|, 0)}=e^{V \Sigma(m \cos \theta-|m|)} .
$$

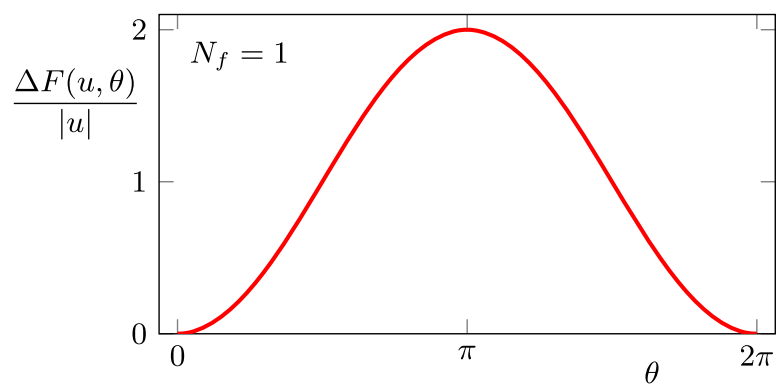

FIG. 3. Difference of the free energies of the phase-quenched and the full theory for one-flavor QCD [see Eq. (15)] divided by the absolute value of the rescaled quark mass. Here $u>0$.

Therefore exponential cancellations take place at $\theta \neq 0$. The function $\Delta F(m, \theta)=V \Sigma|m|(1-\operatorname{sign}(m) \cos \theta)$ is shown in Fig. 3 for $m>0$. In agreement with the general argument above, it assumes its maximum at $\cos \theta=-\operatorname{sign}(m)$, where the sign problem is most severe, while the sign problem is absent for $\cos \theta=\operatorname{sign}(m)$. The free energy is a smooth function of the quark mass and the $\theta$ angle, which reflects the fact that the one-flavor theory has no phase transition.

For two flavors the ratio is given by

$$
\exp \left[-\Delta F\left(m_{1}, m_{2}, \theta\right)\right]=\frac{Z\left(m_{1}, m_{2}, \theta\right)}{Z\left(\left|m_{1}\right|,\left|m_{2}\right|, 0\right)} .
$$

The $\varepsilon$-domain result for the two-flavor partition function is given by the integral over $\mathrm{SU}(2)$ in Eq. (9). In terms of the microscopic variables $u_{k}=m_{k} V \Sigma$ it becomes [5]

$$
Z\left(u_{1}, u_{2}, \theta\right)=\frac{2 I_{1}\left(\sqrt{u_{1}^{2}+u_{2}^{2}+2 u_{1} u_{2} \cos \theta}\right)}{\sqrt{u_{1}^{2}+u_{2}^{2}+2 u_{1} u_{2} \cos \theta}}
$$

with $I_{k}$ the modified Bessel function of the first kind. Again the difference $\Delta F$ assumes its maximum at $\cos \theta=$ $-\operatorname{sign}\left(m_{1} m_{2}\right)$; see the plots in Fig. 4. Therefore the sign problem is most severe either at $\theta=0$ when both masses have opposite signs or at $\theta=\pi$ when both masses have the
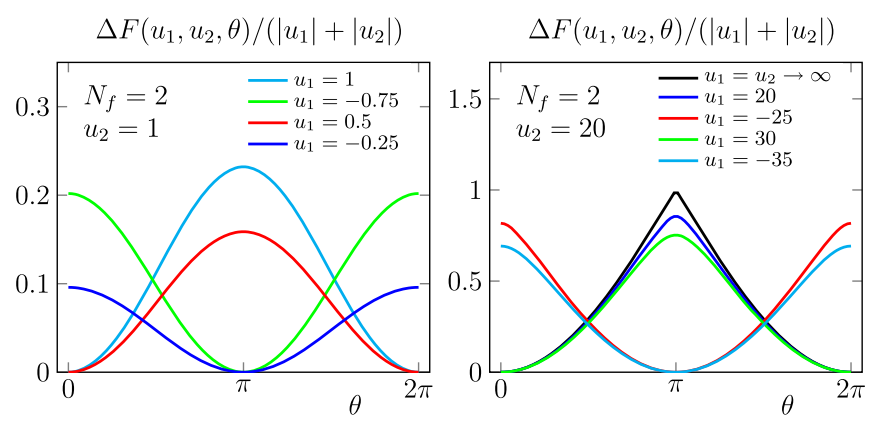

FIG. 4. Free-energy difference as a function of $\theta$ for two flavors and several quark masses. The functions are normalized by the sum of the quark masses so that the large-mass limit remains finite. The black curve in the plot on the right is the thermodynamic limit for equal quark masses; see Eq. (20). 
same sign. This can also be seen in the exponentially increasing oscillations of the level density when increasing the $\theta$ angle; see Fig. 9 below. The sign problem is absent only for $\cos \theta=\operatorname{sign}\left(m_{1} m_{2}\right)$.

We illustrate the free-energy difference in Fig. 4. The partition function (17) is monotonically decreasing (increasing) in $\theta \in[0, \pi]$ for quark masses of equal (opposite) signs. This carries over to an increase (decrease) of the free energy. The free-energy difference is also strictly increasing (decreasing) with respect to the moduli of the masses for equal (opposite) signs. For equal masses it simplifies to

$$
\Delta F(u, u, \theta)=\log \left[\frac{I_{1}(2 u) \cos (\theta / 2)}{I_{1}(2 u \cos (\theta / 2))}\right],
$$

where the monotonicity can be checked easily.

The free-energy difference has the thermodynamic limit

$$
\begin{aligned}
& \lim _{\left|u_{1}\right|,\left|u_{2}\right| \gg 1} \Delta F\left(u_{1}, u_{2}, \theta\right) \\
& =\left|u_{1}\right|+\left|u_{2}\right|-\sqrt{u_{1}^{2}+u_{2}^{2}+2 u_{1} u_{2} \cos \theta},
\end{aligned}
$$

where only the Bessel functions had to be approximated. (There are subleading logarithmic corrections to this result.) For equal masses the thermodynamic limit becomes

$$
\lim _{|u| \gg 1} \Delta F(u, u, \theta)=2|u|(1-|\cos (\theta / 2)|),
$$

which has a kink at the Dashen point $\theta=\pi$.

\section{DECOMPOSITION OF THE SPECTRAL DENSITY AND THE CHIRAL CONDENSATE}

In the limit of zero quark masses, the chiral condensate at fixed topological charge has a discontinuity on the support of the Dirac spectrum, which is dense in the thermodynamic limit. We have to understand how this discontinuity can be moved away from the support of the spectrum at nonzero $\theta$ angle. When the spectral density is positive, this is certainly not possible. However, the averaging procedure to obtain the spectral density includes the fermion determinant. This determinant is not positive definite at nonzero $\theta$ angle, and thus the spectral density is generically not positive definite. Moreover, the spectral density is normalized with respect to the partition function at $\theta \neq 0$, which is exponentially smaller than the phase-quenched partition function, and therefore may result in a spectral density that increases exponentially with the volume. Indeed, we know from QCD at nonzero chemical potential [17] and from QCD-like theories with indefinite measure [18] that the discontinuity of the chiral condensate can be shifted when the spectral density oscillates with an amplitude that is exponentially large in $V$ and with a period that scales as
$1 / V$. As a first step toward understanding this behavior, we decompose the spectral density and the chiral condensate into various contributions.

We denote the spectral density of the Dirac operator at fixed topological charge $\nu$ by $\rho_{\nu}(\lambda, M)$. The spectral density at fixed $\theta$ is defined by

$$
\rho(\lambda, M, \theta)=\sum_{\nu} P_{\nu}(M, \theta) \rho_{\nu}(\lambda, M),
$$

where $P_{\nu}$ is the statistical weight to find a gauge-field configuration with topological charge $\nu$,

$$
P_{\nu}(M, \theta)=\frac{Z_{\nu}(M) e^{i \nu \theta}}{\sum_{\nu} Z_{\nu}(M) e^{i \nu \theta}}=\frac{Z_{\nu}(M) e^{i \nu \theta}}{Z(M, \theta)} .
$$

To obtain a more detailed picture we split the spectral density into a zero-mode part and a nonzero-mode part,

$$
\rho(\lambda, M)=\rho^{\mathrm{zm}}(\lambda, M)+\rho^{\mathrm{nzm}}(\lambda, M) .
$$

This splitting is valid both at fixed $\nu$ and at fixed $\theta$. Equation (21) holds separately for the zero-mode and nonzero-mode parts.

The zero-mode part of the density at fixed $\nu$ is

$$
\rho_{\nu}^{\mathrm{zm}}(\lambda)=|\nu| \delta(\lambda) .
$$

We will see in the next section that $\rho^{\mathrm{zm}}$ gives a contribution to the chiral condensate that diverges exponentially if a sign problem is present. This contribution must be canceled by a similar contribution of the nonzero-mode part $\rho^{\text {nzm }}$ to obtain a finite condensate. The question is what part of $\rho^{\mathrm{nzm}}$ is responsible for this cancellation. Obviously there is no unique answer to this question, but we know that this cancellation also has to take place in the quenched approximation. Therefore we decompose $\rho^{\mathrm{nzm}}$ at fixed $\nu$ into a quenched part (obtained by setting $N_{f}=0$ ) and a dynamical part (the remainder),

$$
\rho_{\nu}^{\mathrm{nzm}}(\lambda, M)=\rho_{\nu}^{q}(\lambda)+\rho_{\nu}^{d}(\lambda, M) .
$$

The $\varepsilon$-domain result for the quenched part in terms of the microscopic variable $x=\lambda V \Sigma$ reads [22]

$$
\rho_{\nu}^{q}(x)=\frac{|x|}{2}\left[J_{\nu}^{2}(x)-J_{\nu+1}(x) J_{\nu-1}(x)\right]
$$

with $J_{k}$ the Bessel function of the first kind. The dynamical part depends, in addition to $x$, also on the quark masses, which on the microscopic scale we collect in $\vec{u}=$ $\left(u_{1}, \ldots, u_{N_{f}}\right)$. Explicit expressions can be found in [23,24].

An alternative to Eq. (25) would be to split $\rho^{\text {nzm }}$ at fixed $\theta$ into a phase-quenched part [12], obtained by letting $M \rightarrow|M|$ and $\theta \rightarrow 0$, and an oscillating remainder. The cancellation is then achieved by the phase-quenched part, 
but this part is more complicated than the quenched part, and therefore we do not consider it in this paper.

The chiral condensate is obtained from the spectral density by the relation

$$
\Sigma(m, M)=\frac{1}{V} \int_{-\infty}^{\infty} d \lambda \frac{\rho(\lambda, M)}{i \lambda+m},
$$

where $m$ is a valence quark mass. It is convenient to distinguish $m$ from the sea quark masses in $M$, but at the end of the calculation $m$ will usually be set equal to one of the sea quark masses. Again, Eq. (27) holds both at fixed $\nu$ and at fixed $\theta$. On the macroscopic scale Eq. (27) requires regularization, but in the $\varepsilon$ domain it is valid as it stands.

We now split the condensate into zero-mode, quenched, and dynamical parts, obtained by replacing $\rho$ in Eq. (27) by $\rho^{\mathrm{zm}}, \rho^{q}$, and $\rho^{d}$, respectively. This leads to

$$
\Sigma(m, M)=\Sigma^{\mathrm{zm}}(m, M)+\Sigma^{q}(m, M)+\Sigma^{d}(m, M)
$$

at either fixed $\nu$ or fixed $\theta$. As in Eq. (21) we have

$$
\Sigma(m, M, \theta)=\sum_{\nu} P_{\nu}(M, \theta) \Sigma_{\nu}(m, M),
$$

and this holds separately for all three contributions.

All equations in this section can be translated to the microscopic scale by the replacements $\lambda=x / V \Sigma$ and $M=\vec{u} / V \Sigma$. In Eq. (27), the prefactor $1 / V$ is then replaced by $\Sigma$. For the valence mass on the microscopic scale we will use the notation $\hat{u}=m V \Sigma$.

\section{CANCELLATION OF ZERO-MODE AND QUENCHED CONTRIBUTIONS}

In this section we show, for any number of flavors, that the exponentially increasing contribution of the zero modes to the chiral condensate is canceled by an exponentially increasing contribution from the quenched part of the spectrum. The expressions for the contribution of the zero modes are valid without any assumptions, but the other calculations are performed in the $\varepsilon$ domain of QCD.

Using Eqs. (21) and (24), the zero-mode part of the microscopic spectral density at fixed $\theta$ is given by

$$
\rho^{\mathrm{zm}}(x, \vec{u}, \theta)=\frac{\delta(x)}{Z(\vec{u}, \theta)} \sum_{\nu} e^{i \nu \theta}|\nu| Z_{\nu}(\vec{u}) .
$$

Using the Fourier transform of Eq. (4),

$$
Z_{\nu}(\vec{u})=\int_{-\pi}^{\pi} \frac{d \theta}{2 \pi} e^{-i \nu \theta} Z(\vec{u}, \theta),
$$

the sum on the right-hand side (RHS) can be rewritten in the form $\sum_{\nu} e^{i \nu \theta}|\nu| Z_{\nu}(\vec{u})=\int_{-\pi}^{\pi} \frac{d \varphi}{2 \pi} A(\varphi)[Z(\vec{u}, \theta-\varphi)-Z(\vec{u}, \theta)]$,

where $A(\varphi)$ is the Fourier transform

$$
A(\varphi)=\sum_{\nu} e^{i \nu \varphi}|\nu|
$$

and the symbol $f$ stands for a principal-value integral. Several comments are in order. (i) The definition (33) is to be understood as a distribution acting on test functions that are twice differentiable and vanish at $\varphi=0$, where the sum over $\nu$ is divergent. We always assume $\varphi \in[-\pi, \pi]$ because of the integral (32). (ii) Since the integral over $A(\varphi)$ vanishes, we could subtract $Z(\vec{u}, \theta)$ in (32) to end up with a test function that indeed vanishes at $\varphi=0$. (iii) In the following we always deal with test functions that are twice differentiable and vanish at $\varphi=0$. This justifies the introduction of a regulator $\varepsilon>0$ which results in a sum that is pointwise convergent except for $\varphi=0$,

$$
\begin{aligned}
A(\varphi) & =\lim _{\varepsilon \rightarrow 0} \sum_{\nu=-\infty}^{\infty}|\nu| e^{i \nu \varphi-|\nu| \varepsilon} \\
& =\lim _{\varepsilon \rightarrow 0} \sum_{\nu=1}^{\infty} \nu\left(e^{-(\varepsilon+i \varphi) \nu}+e^{-(\varepsilon-i \varphi) \nu}\right) \\
& =\lim _{\varepsilon \rightarrow 0} \frac{\partial}{\partial \varepsilon} \frac{e^{-\varepsilon}-\cos \varphi}{\cosh \varepsilon-\cos \varphi} \\
& =-\frac{1}{2 \sin ^{2} \frac{\varphi}{2}}(\text { valid for } \varphi \neq 0) .
\end{aligned}
$$

Note that due to the pointwise convergence, the integral of $A(\varphi)$ in the form (34) no longer vanishes so that the subtractions have to be made before the limit $\varepsilon \rightarrow 0$ is taken. For a function $f$ that is twice differentiable at zero we therefore have

$f_{-\pi}^{\pi} d \varphi A(\varphi)[f(\varphi)-f(0)]=-f_{-\pi}^{\pi} d \varphi \frac{f(\varphi)-f(0)}{2 \sin ^{2} \frac{\varphi}{2}}$.

Finally, we note that $-1 / 2 \sin ^{2} \frac{\varphi}{2}=\partial_{\varphi} \cot \frac{\varphi}{2}$ is a total derivative which can be integrated by parts on test functions that vanish at $\varphi=0$.

The zero-mode part of the spectral density thus becomes

$\rho^{\mathrm{zm}}(x, \vec{u}, \theta)=\delta(x) \int_{-\pi}^{\pi} \frac{d \varphi}{2 \pi} \frac{1}{2 \sin ^{2} \frac{\varphi}{2}}\left[1-\frac{Z(\vec{u}, \theta-\varphi)}{Z(\vec{u}, \theta)}\right]$.

The zero-mode contribution to the chiral condensate follows trivially from Eq. (27):

$\frac{\Sigma^{\mathrm{zm}}(\hat{u}, \vec{u}, \theta)}{\Sigma}=\frac{1}{\hat{u}} \int_{-\pi}^{\pi} \frac{d \varphi}{2 \pi} \frac{1}{2 \sin ^{2} \frac{\varphi}{2}}\left[1-\frac{Z(\vec{u}, \theta-\varphi)}{Z(\vec{u}, \theta)}\right]$. 
In the derivation of this formula we did not make any assumptions on the scaling behavior of the quark masses, and we stress that this result is valid in general.

From the discussion of the sign problem in Sec. III we know that the ratio $Z(\vec{u}, \theta) / Z(|\vec{u}|, 0)$ takes its maximum at $\cos \theta=\operatorname{sign} \operatorname{det} M$. Hence, for $\operatorname{det} M>0$, the integral (37) is dominated by the region around $\varphi=\theta$, and for $\operatorname{det} M<0$ it is dominated by the region around $\varphi=\theta-\pi$. For $\cos \theta \neq \operatorname{sign} \operatorname{det} M$, the partition function $Z(\vec{u}, \theta)$ is exponentially smaller (in $\vec{u}$ ) than $Z(|\vec{u}|, \theta=0$ ), resulting in a contribution to the chiral condensate that increases exponentially with the volume.

The quenched part of the spectral density at fixed $\theta$ is

$$
\begin{aligned}
\rho^{q}(x, \vec{u}, \theta) & =\frac{1}{Z(\vec{u}, \theta)} \sum_{\nu} e^{i \nu \theta} \rho_{\nu}^{q}(x) Z_{\nu}(\vec{u}) \\
& =\int_{-\pi}^{\pi} \frac{d \varphi}{2 \pi} \tilde{\rho}^{q}(x, \varphi) \frac{Z(\vec{u}, \theta-\varphi)}{Z(\vec{u}, \theta)}
\end{aligned}
$$

with

$$
\tilde{\rho}^{q}(x, \varphi)=\sum_{\nu} e^{i \nu \varphi} \rho_{\nu}^{q}(x)=\frac{J_{1}\left(2|x| \sin \frac{\varphi}{2}\right)}{2 \sin \frac{\varphi}{2}} .
$$

The last equality in Eq. (39) was obtained from Eq. (26) using Eq. (A7). To avoid confusion, we note that $\tilde{\rho}^{q}(x, \theta)$ is the spectral density of the true quenched theory at fixed $\theta$. It is convoluted with $Z(\vec{u}, \theta-\varphi) / Z(\vec{u}, \varphi)$ as shown in Eq. (38) to obtain what we have defined as the quenched part of the spectral density. The convolution introduces the quark masses into $\rho^{q}(x, \vec{u}, \theta)$, while $\tilde{\rho}^{q}(x, \varphi)$ is independent of the quark masses.

Equation (38) results in the "quenched" contribution to the chiral condensate

$$
\begin{aligned}
& \frac{\Sigma^{q}(\hat{u}, \vec{u}, \theta)}{\Sigma} \\
& =\int_{0}^{\infty} \frac{2 d x \hat{u}}{x^{2}+\hat{u}^{2}} \int_{-\pi}^{\pi} \frac{d \varphi}{2 \pi} \frac{J_{1}\left(2 x \sin \frac{\varphi}{2}\right)}{2 \sin \frac{\varphi}{2}} \frac{Z(\vec{u}, \theta-\varphi)}{Z(\vec{u}, \theta)} \\
& =\int_{-\pi}^{\pi} \frac{d \varphi}{2 \pi}\left[\frac{1}{2 \hat{u} \sin ^{2} \frac{\varphi}{2}}-\frac{K_{1}\left(2\left|\hat{u} \sin \frac{\varphi}{2}\right|\right)}{\operatorname{sign}(\hat{u})\left|\sin \frac{\varphi}{2}\right|}\right] \frac{Z(\vec{u}, \theta-\varphi)}{Z(\vec{u}, \theta)} .
\end{aligned}
$$

In the last line we have used Eq. (C8) to obtain the modified Bessel function of the second kind $K_{1}$. Note that the poles at $\varphi=0$ cancel so that the integral can be evaluated as an ordinary integral. Also for the quenched contribution, the integral is dominated by the region around $\varphi=\theta$ or $\varphi=$ $\theta-\pi$ (depending on the sign of the product of the quark masses) where the effective $\theta$ angle vanishes. For $\theta+\arg \operatorname{det} M \neq 0$, this again leads to contributions that increase exponentially. For $N_{f}=0$ the ratio of partition functions in Eq. (40) is equal to unity. The integral over $\varphi$ of the expression in square brackets is equal to the "quenched" part of the condensate at topological charge zero, i.e., $\Sigma_{\nu=0}^{q}(\hat{u})$, which follows by writing the RHS of Eq. (39) as a Fourier sum.

We now show that the two exponentially increasing contributions to the chiral condensate cancel. The sum of zero-mode and quenched contributions is given by

$$
\begin{aligned}
& \frac{\Sigma^{\mathrm{zm}}(\hat{u}, \vec{u}, \theta)}{\Sigma}+\frac{\Sigma^{q}(\hat{u}, \vec{u}, \theta)}{\Sigma} \\
& =\int_{-\pi}^{\pi} \frac{d \varphi}{2 \pi}\left[\frac{1}{2 \hat{u} \sin ^{2} \frac{\varphi}{2}}-\frac{K_{1}\left(2\left|\hat{u} \sin \frac{\varphi}{2}\right|\right)}{\operatorname{sign}(\hat{u})\left|\sin \frac{\varphi}{2}\right|} \frac{Z(\vec{u}, \theta-\varphi)}{Z(\vec{u}, \theta)}\right] \\
& =\frac{\sum_{\nu=0}^{q}}{\Sigma}-f_{-\pi}^{\pi} \frac{d \varphi}{2 \pi} \frac{K_{1}\left(2\left|\hat{u} \sin \frac{\varphi}{2}\right|\right)}{\operatorname{sign}(\hat{u})\left|\sin \frac{\varphi}{2}\right|}\left[\frac{Z(\vec{u}, \theta-\varphi)}{Z(\vec{u}, \theta)}-1\right] .
\end{aligned}
$$

This integral converges as a principal-value integral.

The partition function is given in Eq. (9). We write $U \in$ $\mathrm{SU}\left(N_{f}\right)$ in the form $U=V \operatorname{diag}\left(e^{i \varphi_{1}}, \ldots, e^{i \varphi_{N_{f}}}\right) V^{\dagger}$ with $V \in \mathrm{U}\left(N_{f}\right) / \mathrm{U}(1)^{N_{f}}$ and $\sum_{k} \varphi_{k}=0$ and perform a change of variables from $U$ to $V$ and $\vec{\varphi}=\left(\varphi_{1}, \ldots, \varphi_{N_{f}}\right)$. The Jacobian of this transformation is independent of $V$. If we assume degenerate quark masses, the integrand is also independent of $V$ so that the integration over $V$ simply gives an irrelevant constant. For nondegenerate quark masses the integral over $V$ is of the Harish-ChandraItzykson-Zuber type [25] and leads to

$$
\begin{aligned}
Z(\vec{u}, \theta) \sim & \int \prod_{k} \frac{d \varphi_{k}}{2 \pi}\left|\Delta\left(e^{i \vec{\varphi}}\right)\right|^{2} \delta\left(\sum_{k} \varphi_{k}\right) \\
& \times \frac{\operatorname{det}\left[\exp \left[u_{k} \cos \left(\varphi_{\ell}+\theta / N_{f}\right)\right]\right]}{\Delta(\vec{u}) \Delta\left(\cos \left(\vec{\varphi}+\theta / N_{f}\right)\right)},
\end{aligned}
$$

where $\Delta(\vec{x})=\prod_{a<b}\left(x_{b}-x_{a}\right)$ denotes the Vandermonde determinant. Unless indicated otherwise, a product or a sum over $k$ is understood to run from 1 to $N_{f}$. The symbol $\sim$ indicates that we have suppressed the normalization constant.

The integrand in (42) is symmetric under permutations of the $\varphi_{k}$. We denote the nonexponential terms by $f(\vec{u}, \vec{\varphi}+$ $\left.\theta / N_{f}\right)=\left|\Delta\left(e^{i \vec{\varphi}}\right)\right|^{2} /\left(\Delta(\vec{u}) \Delta\left(\cos \left(\vec{\varphi}+\theta / N_{f}\right)\right)\right)$ and expand the determinant of $\exp \left[u_{k} \cos \left(\varphi_{\ell}+\theta / N_{f}\right)\right]$ to obtain

$$
\begin{aligned}
Z(\vec{u}, \theta) \sim & \int \prod_{k} \frac{d \varphi_{k}}{2 \pi} f\left(\vec{u}, \vec{\varphi}+\theta / N_{f}\right) \\
& \times e^{\sum_{k} u_{k} \cos \left(\varphi_{k}+\theta / N_{f}\right)} \delta\left(\sum_{k} \varphi_{k}\right) .
\end{aligned}
$$

After shifting each $\varphi_{k}$ by $-\theta / N_{f}$ and performing the integration over $\varphi_{1}$ using the $\delta$ function, Eq. (43) becomes 


$$
\begin{aligned}
Z(\vec{u}, \theta) \sim & \int \prod_{k \geq 2} \frac{d \varphi_{k}}{2 \pi} f(\vec{u}, \overrightarrow{\bar{\varphi}}) \\
& \times \exp \left[u_{1} \cos \left(\theta-\sum_{k \geq 2} \varphi_{k}\right)+\sum_{k \geq 2} u_{k} \cos \varphi_{k}\right],
\end{aligned}
$$

where $\overrightarrow{\bar{\varphi}}=\left(\theta-\sum_{k \geq 2} \varphi_{k}, \varphi_{2}, \ldots, \varphi_{k}\right)$. We will use this representation for the partition function in the denominator of Eq. (41).

Next, we consider the integral in the last line of Eq. (41). For large $\hat{u}$, the Bessel function behaves as

$$
K_{\nu}\left(2\left|\hat{u} \sin \frac{\varphi}{2}\right|\right)=\frac{\sqrt{\pi}}{\sqrt{4\left|\hat{u} \sin \frac{\varphi}{2}\right|}} e^{-2\left|\hat{u} \sin \frac{\varphi}{2}\right|}[1+O(1 / \hat{u})] .
$$

Therefore the -1 term in the last line of Eq. (41) cannot result in exponentially large contributions, but this term regularizes the integral at $\varphi=0$.

We now combine Eq. (43) with $\theta$ replaced by $\theta-\varphi$ and Eq. (45) and consider the contribution to the chiral condensate that gives the exponentially large terms. After shifting each $\varphi_{k}$ by $(\varphi-\theta) / N_{f}$, we obtain

$$
\begin{aligned}
& \frac{1}{Z(\vec{u}, \theta)} \int \frac{d \varphi}{2 \pi} \int \prod_{k} \frac{d \varphi_{k}}{2 \pi} \delta\left(\sum_{k} \varphi_{k}-\theta+\varphi\right) f(\vec{u}, \vec{\varphi}) \\
& \quad \times \exp \left[-2\left|\hat{u} \sin \frac{\varphi}{2}\right|+\sum_{k} u_{k} \cos \varphi_{k}\right] \\
& =\frac{1}{Z(\vec{u}, \theta)} \int \prod_{k} \frac{d \varphi_{k}}{2 \pi} f(\vec{u}, \vec{\varphi}) \\
& \quad \times \exp \left[-2\left|\hat{u} \sin \frac{1}{2}\left(\theta-\sum_{k} \varphi_{k}\right)\right|+\sum_{k} u_{k} \cos \varphi_{k}\right] .
\end{aligned}
$$

We now set the valence mass $\hat{u}$ equal to one of the sea quark masses, say $u_{1}$. Using the trigonometric identity $\cos \alpha-\cos \beta=2 \sin [(\beta+\alpha) / 2] \sin [(\beta-\alpha) / 2]$, the exponent of the last line in (46) can be written as

$$
\begin{aligned}
& -2\left|u_{1} \sin \frac{\theta-\sum_{k} \varphi_{k}}{2}\right|+u_{1} \cos \varphi_{1}-u_{1} \cos \left(\theta-\sum_{k \geq 2} \varphi_{k}\right) \\
& +u_{1} \cos \left(\theta-\sum_{k \geq 2} \varphi_{k}\right)+\sum_{k \geq 2} u_{k} \cos \varphi_{k} \\
& =-2\left|u_{1} \sin \frac{\theta-\sum_{k} \varphi_{k}}{2}\right|+2 u_{1} \sin \frac{\theta+\varphi_{1}-\sum_{k \geq 2} \varphi_{k}}{2} \\
& \quad \times \sin \frac{\theta-\sum_{k} \varphi_{k}}{2}+u_{1} \cos \left(\theta-\sum_{k \geq 2} \varphi_{k}\right)+\sum_{k \geq 2} u_{k} \cos \varphi_{k} \\
& \leq u_{1} \cos \left(\theta-\sum_{k \geq 2} \varphi_{k}\right)+\sum_{k \geq 2} u_{k} \cos \varphi_{k},
\end{aligned}
$$

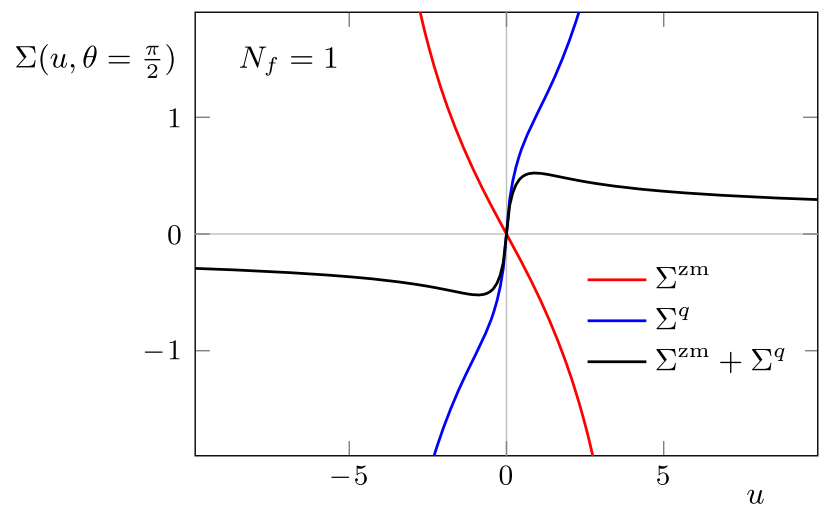

FIG. 5. Zero-mode (red curve) and quenched (blue curve) contribution to the chiral condensate for $N_{f}=1$ and $\theta=\pi / 2$ as a function of the rescaled quark mass. Each contribution increases exponentially, but their sum (black curve) remains finite.

where in the last line we used the fact that the absolute value of the second term is always smaller than the absolute value of the first term. The RHS of this estimate is exactly the exponent of the integrand for $Z(\vec{u}, \theta)$; cf. (44).

We have thus found that in Eq. (41) the exponent of the numerator is always smaller than or equal to the exponent of the denominator. Therefore the sum of the zero-mode and quenched contributions to the chiral condensate does not increase exponentially for large rescaled masses $u_{k}$. The preexponential terms may have (even strong) effects on the integral. They can be zero, can diverge at the saddle points, or can prevent us from reaching some of the saddle points. However, none of these effects can lead to an exponential increase because the integral in (41) is well defined and finite at fixed $\vec{u}$.

The exponential cancellation is illustrated in Fig. 5 for $N_{f}=1$ at $\theta=\pi / 2$ and in Fig. 6 for $N_{f}=2$ at $\theta=0$, where both $\Sigma_{1}^{\mathrm{zm}}$ and $\Sigma_{1}^{q}$ increase exponentially in the quadrants where $u_{1} u_{2}<0$. The sum of the two contributions remains finite. Also shown in Fig. 6 is the dynamical part of the chiral condensate; see Eq. (82).

Finally, we give a heuristic argument why the cancellation between the zero-mode contribution and the quenched contribution to the chiral condensate takes place. When we have $|\nu|$ zero modes, the nonzero eigenvalues on the microscopic scale are, on average, shifted from the origin by $|\nu|$; see Ref. [26] [Sec. VIII A]. To find a definitive gap one needs to assume $1 \ll \nu \ll V \Lambda_{\mathrm{QCD}}^{4}$. Explicitly, one can easily show that the asymptotic behavior of the microscopic quenched density is given by

$$
\rho_{\nu}^{q}(x) \stackrel{|\nu| \gg 1}{\approx} \frac{1}{\pi} \frac{\sqrt{x^{2}-\nu^{2}}}{|x|} \theta(|x|-|\nu|) .
$$

The corresponding chiral condensate $\Sigma_{\nu}^{q}(\hat{u})$ can be calculated along Eq. (27), i.e., 


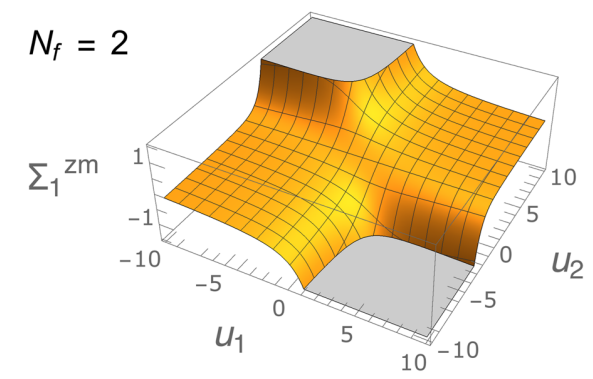

$$
\begin{gathered}
\frac{\sum_{\nu}^{q}(\hat{u})}{\Sigma} \stackrel{|\nu| \gg 1}{\approx} \frac{2 \hat{u}}{\pi} \int_{|\nu|}^{\infty} d x \frac{\sqrt{x^{2}-\nu^{2}}}{x\left(x^{2}+\hat{u}^{2}\right)} \\
=\frac{|\nu|}{\hat{u}}\left(\sqrt{1+\frac{\hat{u}^{2}}{\nu^{2}}}-1\right) .
\end{gathered}
$$

For large masses at large, fixed topological index, the quenched chiral condensate has the approximate form

$$
\frac{\sum_{\nu}^{q}(\hat{u})}{\Sigma} \stackrel{|\hat{u}| \gg|\nu| \gg 1}{\approx} \operatorname{sign}(\hat{u})-\frac{|\nu|}{\hat{u}} .
$$

The second term shows that the nonzero-mode spectrum has been depleted by $|\nu|$ modes so that the total number of eigenvalues does not depend on $\nu$. The contribution of the zero modes is given by $\Sigma_{\nu}^{\mathrm{zm}}(\hat{u})=|\nu| / \hat{u}$.

At fixed (large) $\nu$ we thus find that for large $\hat{u}$ the $1 / \hat{u}$ contribution to the quenched part of the chiral condensate exactly cancels the contribution from the zero modes. At fixed $\theta$ the contribution of the fermion determinant leads to exponentially large terms (instead of $1 / \hat{u}$ terms) at large $\hat{u}$, but as shown in Sec. V we again have a cancellation between the zero-mode and quenched parts. This cancellation is deeply rooted in topology and spectral flow, which guarantee that the total number of eigenvalues around zero remains the same. For chiral random matrix theory, this can be shown at the technical level [26], but the argument is much more general: the Dirac spectrum near zero is depleted by exactly the same number of levels as we have zero modes. One could argue that the spectral density at fixed $\theta$ angle mainly involves very large $\nu$ so that in the thermodynamic limit the spectrum acquires a gap at zero. However, since the topological susceptibility is finite in the thermodynamic limit, the number of zero modes is of order $\sqrt{V}$, while the eigenvalue density is of order $1 / V$, resulting in a gap with a width of order $1 / \sqrt{V}$.

\section{ONE-FLAVOR QCD}

In this section we derive a number of explicit results for one-flavor QCD. In the first subsection we compute the contributions to the spectral density at fixed $\theta$ angle. In the second subsection we use these results to compute the dynamical contribution to the chiral condensate and show that a mass-independent total chiral condensate is obtained.

\section{A. One-flavor Dirac spectrum at fixed $\boldsymbol{\theta}$ angle}

The one-flavor partition function of QCD in the $\varepsilon$ domain at fixed $\theta$ is given by

$$
Z(u, \theta)=e^{u \cos \theta}
$$

see Eq. (6). At fixed $\nu$ we therefore have from Eq. (31)

$$
Z_{\nu}(u)=\int_{-\pi}^{\pi} \frac{d \theta}{2 \pi} e^{-i \nu \theta+u \cos \theta}=I_{\nu}(u) .
$$


The spectral density at fixed $\theta$ is given by

$$
\rho(x, u, \theta)=\frac{1}{e^{u \cos \theta}} \sum_{\nu} e^{i \nu \theta} I_{\nu}(u) \rho_{\nu}(x, u) .
$$

In the previous section we already obtained the zero-mode part and the quenched part of the spectral density; see Eqs. (36) and (38), respectively. Explicitly, the zero-mode part reads

$$
\begin{aligned}
\rho^{\mathrm{zm}}(x, u, \theta) & =\delta(x) \int_{-\pi}^{\pi} \frac{d \varphi}{2 \pi} \frac{1-e^{u[\cos (\theta-\varphi)-\cos \theta]}}{2 \sin ^{2} \frac{\varphi}{2}} \\
& =\delta(x) u \int_{-\pi}^{\pi} \frac{d \varphi}{2 \pi} e^{u[\cos (\theta-\varphi)-\cos \theta]} \sin (\varphi-\theta) \cot \frac{\varphi}{2},
\end{aligned}
$$

where the second line follows after partial integration. The quenched part is equal to

$\rho^{q}(x, u, \theta)=\int_{-\pi}^{\pi} \frac{d \varphi}{2 \pi} \frac{J_{1}\left(2|x| \sin \frac{\varphi}{2}\right)}{2 \sin \frac{\varphi}{2}} e^{u[\cos (\theta-\varphi)-\cos \theta]}$.

The dynamical part at fixed $\nu$ is given by $[23,24]$

$\rho_{\nu}^{d}(x, u)=\frac{-|x|}{x^{2}+u^{2}}\left[x J_{\nu}(x) J_{\nu+1}(x)+u \frac{I_{\nu+1}(u)}{I_{\nu}(u)} J_{\nu}^{2}(x)\right]$.

After performing the sums over $\nu$ in Eq. (21) with the help of Eq. (A12) we obtain for the dynamical part at fixed $\theta$

$$
\begin{aligned}
& \rho^{d}(x, u, \theta) \\
& =-\frac{|x|}{x^{2}+u^{2}} \int_{-\pi}^{\pi} \frac{d \varphi}{2 \pi} e^{u[\cos (\theta-\varphi)-\cos \theta]} \\
& \quad \times\left[x \sin \frac{\varphi}{2} J_{1}\left(2 x \sin \frac{\varphi}{2}\right)+u \cos (\theta-\varphi) J_{0}\left(2 x \sin \frac{\varphi}{2}\right)\right] .
\end{aligned}
$$

Note that the imaginary part resulting from (A12) is the integral of a total derivative and therefore vanishes. Moreover, the result has to be real since the expression (56) is invariant under $\nu \leftrightarrow-\nu$, which can be shown using the recursion relations $(\mathrm{C} 1)$ and $(\mathrm{C} 2)$ of Bessel functions.

Adding the dynamical part to the quenched part and performing some simplifications, the nonzero-mode part of the density at fixed $\theta$ is given by

$$
\begin{aligned}
\rho^{\mathrm{nzm}}(x, u, \theta)= & \frac{|x|}{x^{2}+u^{2}} \int_{-\pi}^{\pi} \frac{d \varphi}{2 \pi} e^{u[\cos (\theta-\varphi)-\cos \theta]} \\
& \times\left[\left(u^{2}+x^{2} \cos \varphi\right) \frac{J_{1}\left(2 x \sin \frac{\varphi}{2}\right)}{2 x \sin \frac{\varphi}{2}}\right. \\
& \left.-u \cos (\theta-\varphi) J_{0}\left(2 x \sin \frac{\varphi}{2}\right)\right],
\end{aligned}
$$

which for $\theta=0$ was already obtained in [11,12].
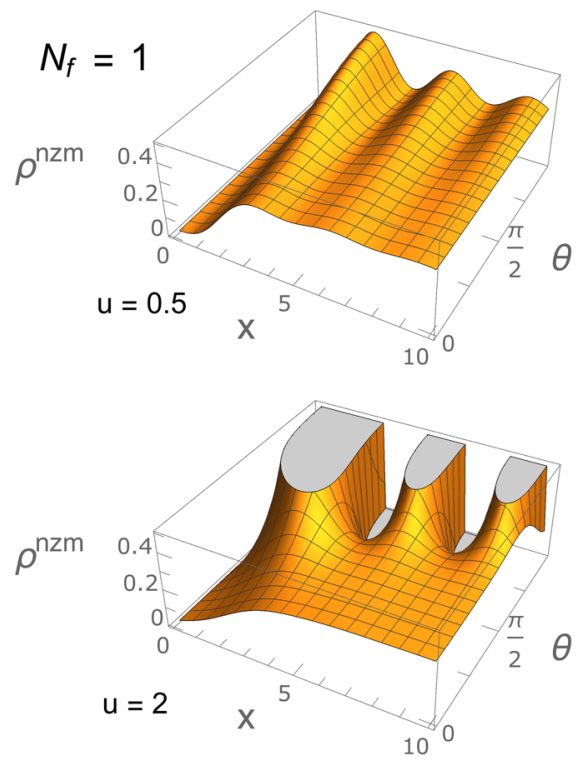

FIG. 7. Microscopic spectral density (58) of the one-flavor theory as a function of the $\theta$ angle for the two rescaled masses $u=0.5$ and $u=2$. The oscillations become stronger with increasing $\theta$ and $u$ and eventually yield an exponentially large spectral density. The reason for this divergence is the nonpositive statistical weight due to the $\theta$ angle.

In Fig. 7 we show the spectral density of the nonzero modes at fixed $\theta$ for rescaled quark masses $u=0.5$ and $u=2$. At nonzero $\theta$ the amplitude of the oscillations increases exponentially with $u$. The only exception is $\theta=\pi$, where the spectral density is well behaved for a large negative mass but increases exponentially with a positive mass $u$; see the discussion prior to Eq. (5).

For large mass $u$, a saddle-point analysis of Eq. (58) shows that the spectral density behaves as

$$
\rho^{\mathrm{nzm}}(x, u, \theta) \stackrel{|u| \gg 1}{\approx} e^{|u|-u \cos \theta} \frac{J_{1}\left(2|x| \sin \frac{\theta_{0}}{2}\right)}{\sqrt{8 \pi|u|} \sin \frac{\theta_{0}}{2}}
$$

with $\theta_{0}=\theta+[1-\operatorname{sign}(u)] \pi / 2$. When the exponent vanishes, i.e., for $\theta=0$ with positive mass or $\theta=\pi$ with negative mass, the asymptotic expansion is still valid, resulting in

$$
\rho^{\mathrm{nzm}}(x, u, 0) \stackrel{|u| \gg 1}{\approx} \frac{|x|}{\sqrt{8 \pi|u|}}
$$

\section{B. Chiral condensate}

In this subsection we use Eq. (27) to compute the chiral condensate for one flavor and show that it is mass independent. Here and in Sec. VIC, the valence quark mass $\hat{u}$ is set equal to the sea quark mass $u$.

The sum of the zero-mode and quenched contributions was already computed in Eq. (41). It is given by 


$$
\begin{aligned}
& \frac{\Sigma^{\mathrm{zm}}(u, \theta)}{\Sigma}+\frac{\Sigma^{q}(u, \theta)}{\Sigma} \\
& =\int_{-\pi}^{\pi} \frac{d \varphi}{2 \pi}\left[\frac{1}{2 u \sin ^{2} \frac{\varphi}{2}}-\frac{K_{1}\left(2\left|u \sin \frac{\varphi}{2}\right|\right)}{\operatorname{sign}(u)\left|\sin \left(\frac{\varphi}{2}\right)\right|} \frac{e^{u \cos (\theta-\varphi)}}{e^{u \cos \theta}}\right] .
\end{aligned}
$$

The contribution from the dynamical part of the density follows from Eqs. (27) and (57) using Eqs. (C10) and (C12),

$$
\begin{aligned}
& \frac{\Sigma^{d}(u, \theta)}{\Sigma}=-2 \int_{-\pi}^{\pi} \frac{d \varphi}{2 \pi} \frac{e^{u \cos (\theta-\varphi)}}{e^{u \cos \theta}}\left[u \sin ^{2} \frac{\varphi}{2} K_{0}\left(2\left|u \sin \frac{\varphi}{2}\right|\right)\right. \\
& \left.+\left|u \sin \frac{\varphi}{2}\right| \cos (\theta-\varphi) K_{1}\left(2\left|u \sin \frac{\varphi}{2}\right|\right)\right] .
\end{aligned}
$$

Since the leading asymptotic behavior of the $K_{\nu}$ Bessel functions [see Eq. (45)] does not depend on the index, we can use the arguments of Sec. V to show that there are no exponentially increasing contributions from the dynamical part. It is noteworthy that this argument holds for an arbitrary number of flavors $N_{f}$. Adding the last two equations we obtain the total chiral condensate

$$
\begin{aligned}
\frac{\Sigma(u, \theta)}{\Sigma}= & f_{-\pi}^{\pi} \frac{d \varphi}{2 \pi}\left\{\frac{1}{2 u \sin ^{2} \frac{\varphi}{2}}-\frac{e^{u \cos (\theta-\varphi)}}{e^{u \cos \theta}}\right. \\
& \times\left[u K_{2}\left(2\left|u \sin \frac{\varphi}{2}\right|\right)-u \cos \varphi K_{0}\left(2\left|u \sin \frac{\varphi}{2}\right|\right)\right. \\
& \left.\left.+2\left|u \sin \frac{\varphi}{2}\right| \cos (\theta-\varphi) K_{1}\left(2\left|u \sin \frac{\varphi}{2}\right|\right)\right]\right\},
\end{aligned}
$$

where we used (C4). Using the recursion relations (C4) and (C5), the Bessel function $K_{2}$ can be rewritten as

$$
\begin{aligned}
K_{2}\left(2\left|u \sin \frac{\varphi}{2}\right|\right) \\
=-\cos \varphi K_{0}\left(2\left|u \sin \frac{\varphi}{2}\right|\right)-\frac{2}{|u|} \operatorname{sign}\left(\sin \frac{\varphi}{2}\right) \\
\times \frac{\partial}{\partial \varphi}\left[\cos \frac{\varphi}{2} K_{1}\left(2\left|u \sin \frac{\varphi}{2}\right|\right)\right] .
\end{aligned}
$$

Then the derivative can be integrated by parts, where the boundary terms of the principal-value integral vanish. Hence we have

$$
\begin{aligned}
\frac{\Sigma(u, \theta)}{\Sigma}= & 2 f_{-\pi}^{\pi} \frac{d \varphi}{2 \pi} \frac{e^{u \cos (\theta-\varphi)}}{e^{u \cos \theta}}\left[u \cos \varphi K_{0}\left(2\left|u \sin \frac{\varphi}{2}\right|\right)\right. \\
& \left.-|u| \operatorname{sign}\left(\sin \frac{\varphi}{2}\right) \sin \left(\theta-\frac{\varphi}{2}\right) K_{1}\left(2\left|u \sin \frac{\varphi}{2}\right|\right)\right] .
\end{aligned}
$$

To show that the principal-value integral is indeed equal to $\cos \theta$ [cf. Eq. (7)], we first note that for $u=0$ we indeed obtain

$$
\frac{\Sigma(u=0, \theta)}{\Sigma}=-f_{-\pi}^{\pi} \frac{d \varphi}{2 \pi} \frac{\sin \left(\theta-\frac{\varphi}{2}\right)}{\sin \frac{\varphi}{2}}=\cos \theta,
$$

where we used the asymptotics $K_{1}(|x|) \approx 1 /|x|$ for $|x| \ll 1$. In the second step we take the mass derivative of the chiral condensate, which is an integral of a total derivative and thus vanishes,

$$
\begin{aligned}
& \frac{d}{d u} \Sigma(u, \theta) \\
& =2 \int_{-\pi}^{\pi} \frac{d \varphi}{2 \pi} \frac{e^{u \cos (\theta-\varphi)}}{e^{u \cos \theta}}\left[\cos \varphi K_{0}\left(2\left|u \sin \frac{\varphi}{2}\right|\right)-2\left|u \sin \frac{\varphi}{2}\right| \cos \varphi K_{1}\left(2\left|u \sin \frac{\varphi}{2}\right|\right)+2 u \sin \frac{\varphi}{2} \sin \left(\theta-\frac{\varphi}{2}\right)\right. \\
& \left.\quad \times K_{0}\left(2\left|u \sin \frac{\varphi}{2}\right|\right)+2 \sin \left(\theta-\frac{\varphi}{2}\right) \sin \frac{\varphi}{2}\left(u \cos \varphi K_{0}\left(2\left|u \sin \frac{\varphi}{2}\right|\right)-|u| \operatorname{sign}\left(\sin \frac{\varphi}{2}\right) \sin \left(\theta-\frac{\varphi}{2}\right) K_{1}\left(2\left|u \sin \frac{\varphi}{2}\right|\right)\right)\right] \\
& =2 \int_{-\pi}^{\pi} \frac{d \varphi}{2 \pi} \frac{d}{d \varphi} \frac{e^{u \cos (\theta-\varphi)}}{e^{u \cos \theta}}\left[\sin \varphi K_{0}\left(2\left|u \sin \frac{\varphi}{2}\right|\right)-2 \operatorname{sign}(u) \sin \theta\left|\sin \frac{\varphi}{2}\right| K_{1}\left(2\left|u \sin \frac{\varphi}{2}\right|\right)\right]=0 .
\end{aligned}
$$

Therefore we conclude that $\Sigma(u, \theta)$ does not depend on $u$ so that

$$
\frac{\Sigma(u, \theta)}{\Sigma}=\cos \theta
$$

The derivation above also shows that the nonzero-mode contribution to the chiral condensate can be simplified to

$$
\frac{\Sigma^{\mathrm{nzm}}(u, \theta)}{\Sigma}=\cos \theta-\frac{\Sigma^{\mathrm{zm}}(u, \theta)}{\Sigma}=\cos \theta-f_{-\pi}^{\pi} \frac{d \varphi}{2 \pi} \frac{e^{u \cos (\theta-\varphi)}}{e^{u \cos \theta}} \sin (\varphi-\theta) \cot \frac{\varphi}{2},
$$

which follows from Eq. (54). 

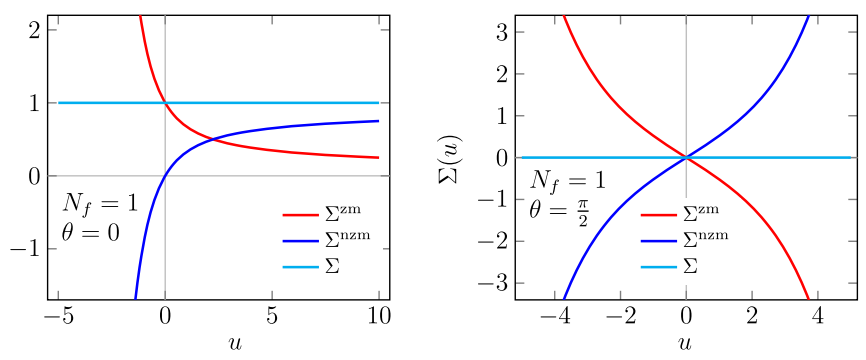

FIG. 8. Chiral condensate for one-flavor QCD and its splitting into the contributions from zero and nonzero modes. Results are given for $\theta=0$ (left) and $\theta=\pi / 2$ (right). While for positive mass and vanishing $\theta$ angle the contributions behave algebraically, they increase exponentially with the volume otherwise, regardless of whether the sign/phase comes from the mass or a nonvanishing $\theta$ angle.

The zero-mode and nonzero-mode contributions to the chiral condensate are plotted as a function of $u$ in Fig. 8 for $\theta=0$ and $\pi / 2$. It becomes obvious that when a nontrivial phase is present, the contributions of both the zero and the nonzero modes grow exponentially with the volume (included in $u$ ), but that they add up to a finite result that gives a mass-independent chiral condensate.

\section{Thermodynamic limit}

We first consider the case when there is no sign problem, i.e., $\cos \theta=\operatorname{sign} u$. For this case the thermodynamic limit $u \rightarrow \infty$ can readily be derived for the zero-mode contribution [5] [Eq. (7.3)],

$$
\begin{aligned}
& \rho^{\mathrm{zm}}(x, u>0, \theta=0)=\delta(x)|u| e^{-|u|}\left[I_{0}(|u|)+I_{1}(|u|)\right] \\
& \approx \delta \mid \gg 1 \\
& \frac{\Sigma^{\mathrm{zm}}(u>0, \theta=0)}{\Sigma} \stackrel{|u| \gg 1}{\mid} \delta \sqrt{\frac{2|u|}{\pi}}, \\
& \frac{2}{\pi|u|} .
\end{aligned}
$$

Therefore the contribution of the zero modes to the chiral condensate behaves like $1 / \sqrt{|u|}$. This can be seen in the left plot of Fig. 8 for positive masses. For $u<0$ and $\theta=\pi$ we find the same asymptotical spectral density as in Eq. (70), but the chiral condensate differs by a minus sign.

We now turn to the general case with a sign problem. In this case we have to perform a saddle-point analysis for the integral determining the level density and the chiral condensate. This is most easily done in Eq. (54). Expanding $\varphi$ about the point where the exponent is maximized, namely $\cos (\varphi-\theta)=\operatorname{sign} u$, we obtain

$$
\begin{aligned}
& \rho^{\mathrm{zm}}(x, u, \theta)=\delta(x) u \Sigma^{\mathrm{zm}}(u, \theta) / \Sigma, \\
& \frac{\Sigma^{\mathrm{zm}}(u, \theta)}{\Sigma} \stackrel{|u| \gg 1}{\approx}-\frac{e^{|u|(1-\operatorname{sign}(u) \cos \theta)}}{\sqrt{2 \pi|u|^{3}}(\operatorname{sign}(u)-\cos \theta)} .
\end{aligned}
$$

Here, we notice the main difference between the signquenched result (70) and the result with a sign problem. The contribution of the zero modes to the chiral condensate vanishes as $1 / \sqrt{|u|}$ in the sign-quenched case, while it diverges exponentially regardless of how small the angle $\theta+\arg u$ is. In particular, the divergence is strongest when $\theta+\arg u= \pm \pi$, which reflects the sign problem observed in the difference of the free energies (15). Additionally, the sign of the contribution of the zero modes to the chiral condensate changes with the sign of the quark mass. Its behavior for $\theta=0$ and $\theta=\pi / 2$ is shown in Fig. 8 .

Whether a sign problem is present or not present, the contribution of the nonzero modes to the chiral condensate is equal to the difference $\Sigma \cos \theta-\Sigma^{\mathrm{zm}}$.

We now consider the thermodynamic limit of the dynamical part $\Sigma^{d}$ [see Eq. (62)], which stays finite for large masses. Its asymptotic behavior is worked out in Appendix D, resulting in

$$
\frac{\Sigma^{d}(u, \theta)}{\Sigma} \stackrel{|u| \gg 1}{\approx} \begin{cases}-\frac{1}{2 u \cos ^{2} \theta}, & u \cos \theta>0 \\ 2 \cos \theta, & u \cos \theta<0 \\ -\frac{\operatorname{sign}(u) \Gamma(5 / 6)}{\sqrt{\pi}|u|^{1 / 3}}, & u \cos \theta=0\end{cases}
$$

where $\Gamma(5 / 6)$ in the last case denotes the $\Gamma$ function.

Finally, to identify the thermodynamic limit of the quenched contribution we can combine the relation $\Sigma(u, \theta)=\Sigma^{\mathrm{zm}}(u, \theta)+\Sigma^{q}(u, \theta)+\Sigma^{d}(u, \theta)=\Sigma \cos \theta$ with the results (70) through (72).

\section{TWO-FLAVOR QCD}

In the first subsection we compute the two-flavor spectral density at fixed $\theta$ angle, which is used in the second subsection to compute the various contributions to the chiral condensate.

\section{A. Two-flavor Dirac spectrum at fixed $\boldsymbol{\theta}$ angle}

The spectral density at fixed $\theta$ angle is again given by Eqs. (21) and (22). The two-flavor partition function at fixed $\theta$ angle has already been given in (17). At fixed topological charge $\nu$ it can be written as [27]

$$
Z_{\nu}(\vec{u})=2 \frac{u_{1} I_{\nu+1}\left(u_{1}\right) I_{\nu}\left(u_{2}\right)-u_{2} I_{\nu+1}\left(u_{2}\right) I_{\nu}\left(u_{1}\right)}{u_{1}^{2}-u_{2}^{2}} .
$$

The zero-mode contribution to the spectral density is determined by Eq. (36) with $Z(\vec{u}, \theta-\varphi)$ as in Eq. (17).

Next we evaluate the nonzero-mode contribution to the spectral density. At fixed $\nu$ the spectral density is given by $[23,24]$ 


$$
\rho_{\nu}(x, \vec{u})=\frac{|x|}{Z_{\nu}(\vec{u})\left(x^{2}+u_{1}^{2}\right)\left(x^{2}+u_{2}^{2}\right)\left(u_{2}^{2}-u_{1}^{2}\right)}\left|\begin{array}{cccc}
J_{\nu-1}(x) / x & J_{\nu}(x) & I_{\nu}\left(-u_{1}\right) & I_{\nu}\left(-u_{2}\right) \\
J_{\nu}(x) & x J_{\nu+1}(x) & u_{1} I_{\nu+1}\left(-u_{1}\right) & u_{2} I_{\nu+1}\left(-u_{2}\right) \\
x J_{\nu+1}(x) & x^{2} J_{\nu+2}(x) & u_{1}^{2} I_{\nu+2}\left(-u_{1}\right) & u_{2}^{2} I_{\nu+2}\left(-u_{2}\right) \\
x^{2} J_{\nu+2}(x) & x^{3} J_{\nu+3}(x) & u_{1}^{3} I_{\nu+3}\left(-u_{1}\right) & u_{2}^{3} I_{\nu+3}\left(-u_{2}\right)
\end{array}\right|,
$$

where $|\cdot|$ denotes the determinant of the matrix. The determinant in Eq. (74) can be rewritten as

$$
\left|\begin{array}{cccc}
x^{-1} J_{\nu-1}(x) & J_{\nu}(x) & I_{\nu}\left(-u_{1}\right) & I_{\nu}\left(-u_{2}\right) \\
J_{\nu}(x) & x J_{\nu+1}(x) & u_{1} I_{\nu+1}\left(-u_{1}\right) & u_{2} I_{\nu+1}\left(-u_{2}\right) \\
-2 J_{\nu}(x) & 0 & \left(u_{1}^{2}+x^{2}\right) I_{\nu}\left(-u_{1}\right) & \left(u_{2}^{2}+x^{2}\right) I_{\nu}\left(-u_{2}\right) \\
-2 x J_{\nu+1}(x) & 0 & u_{1}\left(u_{1}^{2}+x^{2}\right) I_{\nu+1}\left(-u_{1}\right) & u_{2}\left(u_{2}^{2}+x^{2}\right) I_{\nu+1}\left(-u_{2}\right)
\end{array}\right|
$$

by employing the recurrence relations $(\mathrm{C} 1)$ and $(\mathrm{C} 2)$ and the properties of the determinant. In this form the terms involving the upper left $2 \times 2$ block yield the quenched level density (26). The remaining terms represent the dynamical part and can be simplified to

$$
\begin{aligned}
& \rho_{\nu}^{d}(x, \vec{u}) \\
& =-\frac{2|x|}{Z_{\nu}(\vec{u})\left(x^{2}+u_{1}^{2}\right)\left(x^{2}+u_{2}^{2}\right)} \\
& \quad \times\left\{x J_{\nu}(x) J_{\nu+1}(x)\left[u_{1} I_{\nu+1}\left(u_{1}\right) I_{\nu}\left(u_{2}\right)+u_{2} I_{\nu+1}\left(u_{2}\right) I_{\nu}\left(u_{1}\right)\right]\right. \\
& \left.\quad+x^{2} J_{\nu+1}^{2}(x) I_{\nu}\left(u_{1}\right) I_{\nu}\left(u_{2}\right)+u_{1} u_{2} J_{\nu}^{2}(x) I_{\nu+1}\left(u_{1}\right) I_{\nu+1}\left(u_{2}\right)\right\}
\end{aligned}
$$

by employing the symmetry $I_{\nu}(-u)=(-1)^{\nu} I_{\nu}(u)$. Note that the dynamical part $\rho_{\nu}^{d}$ is again symmetric in $\nu \rightarrow-\nu$ because $\rho_{\nu}$ and $\rho_{\nu}^{q}$ are, so that the sum including the phases $e^{i \nu \theta}$ yields a real function.

Now we sum over $\nu$ as shown in (21). The quenched contribution $\rho^{q}$ was already obtained in (38) with the twoflavor partition function $Z\left(u_{1}, u_{2}, \theta\right)$; see Eq. (17). The sum for the dynamical part is more involved, and the required sums over products of four Bessel functions are worked out in Eq. (A13). Adding the quenched contribution, this results in the total spectral density of the nonzero modes,

$$
\begin{aligned}
& \rho^{\mathrm{nzm}}(x, \vec{u}, \theta) \\
& =\frac{|x|}{Z(\vec{u}, \theta)} \int_{-\pi}^{\pi} \frac{d \varphi}{2 \pi}\left[\frac{J_{1}\left(2 x \sin \frac{\varphi}{2}\right)}{2 x \sin \frac{\varphi}{2}} Z(\vec{u}, \theta-\varphi)\right. \\
& \quad+\frac{x\left(2 u_{1} u_{2} e^{i(\varphi-\theta)}+u_{2}^{2}+u_{1}^{2}\right) J_{1}\left(2 x \sin \frac{\varphi}{2}\right)}{\left(x^{2}+u_{1}^{2}\right)\left(x^{2}+u_{2}^{2}\right) i e^{i \frac{\varphi}{2}}} Z(\vec{u}, \theta-\varphi) \\
& \left.\quad-\frac{2\left(u_{1} u_{2} e^{i(\varphi-\theta)}+x^{2} e^{-i \varphi}\right) J_{0}\left(2 x \sin \frac{\varphi}{2}\right)}{\left(x^{2}+u_{1}^{2}\right)\left(x^{2}+u_{2}^{2}\right)} \zeta_{0}(\vec{u}, \theta-\varphi)\right]
\end{aligned}
$$
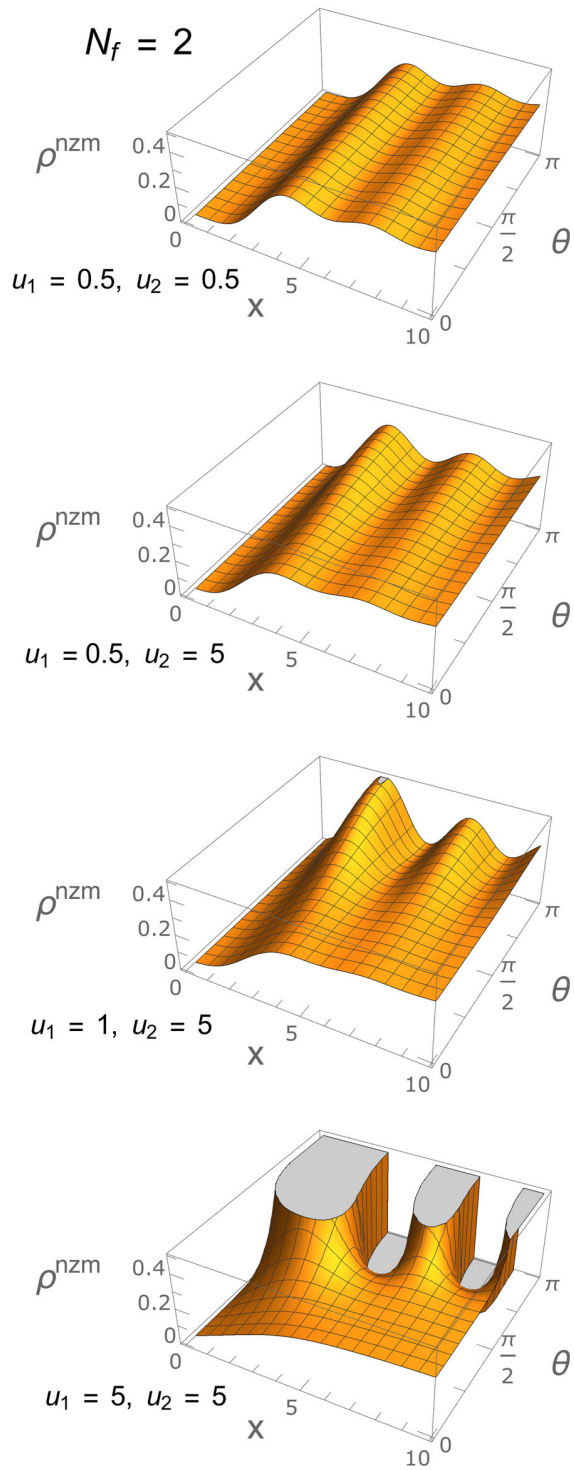

FIG. 9. Microscopic level density for two flavors as a function of the eigenvalue position $x$ and of the $\theta$ angle for various quark masses $u_{1}$ and $u_{2}$. When increasing the quark masses the oscillations become so strong that the level density loses its positivity. 
with

$$
\zeta_{0}(\vec{u}, \alpha)=I_{0}\left(\sqrt{u_{1}^{2}+u_{2}^{2}+2 u_{1} u_{2} \cos \alpha}\right) .
$$

We recognize the first term as the contribution of the quenched part of the spectral density discussed in Sec. V. The imaginary part of the integral vanishes, which follows from the fact that the imaginary part resulting from applying Eq. (A13) yields a total derivative.

The level density of the nonzero modes, $\rho^{\mathrm{nzm}}=\rho^{q}+\rho^{d}$, looks quite complicated. It becomes more presentable when both masses are equal and the $\theta$ angle vanishes,

$$
\begin{aligned}
& \rho^{\mathrm{nzm}}(x, u, u, \theta=0) \\
& =\frac{|x u|}{I_{1}(2|u|)} \int_{-\pi}^{\pi} \frac{d \varphi}{\pi}\left[\frac{J_{1}\left(2 x \sin \frac{\varphi}{2}\right)}{2 x \sin \frac{\varphi}{2}}\right. \\
& \left.\quad \times \frac{I_{1}\left(2 u \cos \frac{\varphi}{2}\right)}{2 u \cos \frac{\varphi}{2}}-\cos \varphi \frac{J_{0}\left(2 x \sin \frac{\varphi}{2}\right) I_{0}\left(2 u \cos \frac{\varphi}{2}\right)}{x^{2}+u^{2}}\right] .
\end{aligned}
$$

The behavior of the level density $\rho^{\text {nzm }}$ is shown in Fig. 9 for various masses. As in the one-flavor case, the amplitude of the level density at nonzero $\theta$ angle increases exponentially with the volume, and its oscillations have a period of $O(1 / V)$. At nonzero $\theta$ angle, oscillations of this type can shift the original discontinuity of the chiral condensate at $m=0$ for fixed $\nu$.

For large mass $u_{2}$ we regain the level density $\rho_{N_{f}=1}^{\mathrm{nzm}}\left(x, u_{1}, \theta\right)$. To obtain this limit we have to approximate the modified Bessel functions by

$I_{\nu}\left(\sqrt{u_{1}^{2}+u_{2}^{2}+2 u_{1} u_{2} \cos \varphi}\right) \approx \frac{e^{\left|u_{2}\right|+\operatorname{sign}\left(u_{2}\right) u_{1} \cos \varphi}}{\sqrt{2 \pi\left|u_{2}\right|}}$.

In contrast, when taking the limit $u_{2} \rightarrow 0$, only the sector of zero topological charge contributes to the partition function, resulting in the spectral density $\rho_{\nu=0}^{\mathrm{nzm}}\left(x, u_{1}, 0\right)$. Because of flavor-topology duality [28] for massless quarks in the microscopic limit (which is most easily understood in terms of the joint eigenvalue distribution of the chiral random matrix theory), this can be written as $\rho_{\nu=N_{f}=1}^{\mathrm{nzm}}\left(x, u=u_{1}\right)$.

\section{B. Chiral condensate}

In this subsection we evaluate the chiral condensate from the spectral density using Eq. (27). We only consider the chiral condensate of the first quark (which we denote by $\Sigma_{1}$ ); i.e., we set $\hat{u}=u_{1}$. The chiral condensate $\Sigma_{2}$ of the second quark can then be obtained by interchanging $u_{1} \leftrightarrow u_{2}$.

The zero-mode and quenched contributions to the chiral condensate are obtained by substituting the two-flavor
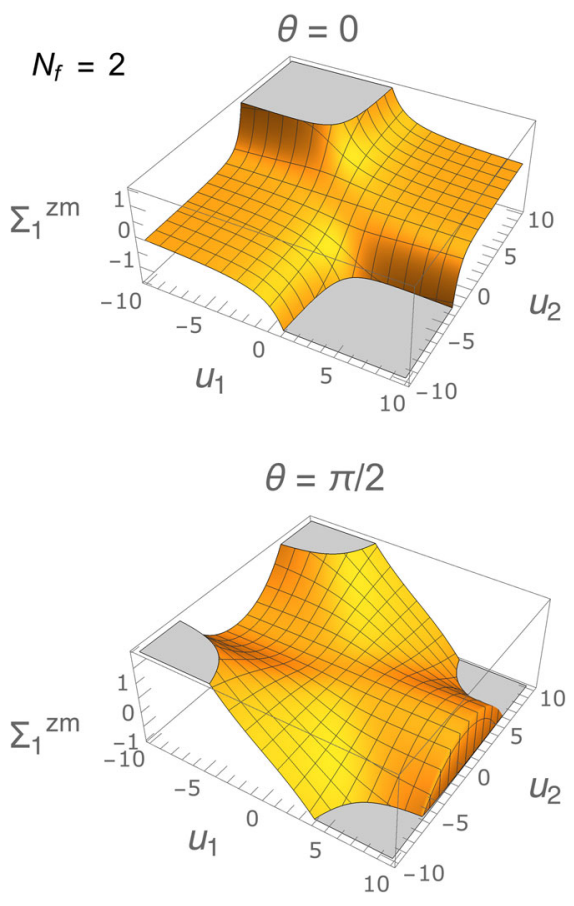

FIG. 10. Zero-mode contribution to the chiral condensate for two flavors as a function of the two quark masses $u_{1}$ and $u_{2}$. For vanishing $\theta$ angle we notice two quadrants where this contribution does not grow exponentially in the masses. For $\theta \in(0, \pi)$ we have only two lines, given by $u_{1} u_{2}=0$, where this contribution remains finite in the limit of large masses.

result (17) for the partition function in Eqs. (37), (40), and (41). We do not repeat the corresponding expressions here. As in the one-flavor case, $\Sigma_{1}^{\mathrm{zm}}(\vec{u}, \theta)$ grows exponentially in the quark masses for $\theta \neq 0$ or for $\theta=0$ and $u_{1} u_{2}<0$. This can be seen nicely in Fig. 10. When the sign problem is absent, $\Sigma_{1}^{\mathrm{zm}}$ remains bounded. For vanishing $\theta$ angle we can use $-1 / 2 \sin ^{2} \frac{\varphi}{2}=\partial_{\varphi} \cot \frac{\varphi}{2}$ and integrate Eq. (37) by parts such that the integral loses its singularity and becomes an ordinary integral,

$$
\begin{aligned}
\frac{\Sigma_{1}^{\mathrm{zm}}(\vec{u}, 0)}{\Sigma}= & \frac{2 u_{2}\left|u_{1}+u_{2}\right|}{I_{1}\left(\left|u_{1}+u_{2}\right|\right)} \int_{-\pi}^{\pi} \frac{d \varphi}{2 \pi} \cos ^{2} \frac{\varphi}{2} \\
& \times \frac{I_{2}\left(\sqrt{u_{1}^{2}+u_{2}^{2}+2 u_{1} u_{2} \cos \varphi}\right)}{u_{1}^{2}+u_{2}^{2}+2 u_{1} u_{2} \cos \varphi} .
\end{aligned}
$$

We employed Eq. (C3) to obtain this result. A similar result can be derived for $\theta=\pi$. When the masses are equal, the integral can be expressed in terms of a hypergeometric function.

The calculation of the dynamical contribution to the chiral condensate is performed in Appendix B and yields 


$$
\begin{aligned}
\frac{\Sigma_{1}^{d}(\vec{u}, \theta)}{\Sigma}= & u_{1} \int_{-\pi}^{\pi} \frac{d \varphi}{\pi}\left\{[ 2 u _ { 1 } u _ { 2 } \operatorname { s i n } \frac { \varphi } { 2 } \operatorname { s i n } ( \frac { \varphi } { 2 } - \theta ) - ( u _ { 1 } ^ { 2 } + u _ { 2 } ^ { 2 } ) \operatorname { s i n } ^ { 2 } \frac { \varphi } { 2 } ] \left[\frac{K_{0}\left(2\left|u_{1} \sin \frac{\varphi}{2}\right|\right)}{u_{2}^{2}-u_{1}^{2}}\right.\right. \\
& \left.+\frac{\left|u_{2}\right| K_{1}\left(2\left|u_{2} \sin \frac{\varphi}{2}\right|\right)-\left|u_{1}\right| K_{1}\left(2\left|u_{1} \sin \frac{\varphi}{2}\right|\right)}{\left(u_{2}^{2}-u_{1}^{2}\right)^{2}\left|\sin \frac{\varphi}{2}\right|}\right] \frac{Z(\vec{u}, \theta-\varphi)}{Z(\vec{u}, \theta)} \\
& +\left[\left|\sin \frac{\varphi}{2}\right| \operatorname{sign}\left(u_{1}\right) \frac{u_{1} \cos \varphi-u_{2} \cos (\theta-\varphi)}{u_{2}^{2}-u_{1}^{2}} K_{1}\left(2\left|u_{1} \sin \frac{\varphi}{2}\right|\right)\right. \\
& \left.\left.+\left(u_{1} u_{2} \cos (\theta-\varphi)-u_{2}^{2} \cos \varphi\right) \frac{K_{0}\left(2\left|u_{1} \sin \frac{\varphi}{2}\right|\right)-K_{0}\left(2\left|u_{2} \sin \frac{\varphi}{2}\right|\right)}{\left(u_{2}^{2}-u_{1}^{2}\right)^{2}}\right] \frac{2 \zeta_{0}(\vec{u}, \theta-\varphi)}{Z(\vec{u}, \theta)}\right\} .
\end{aligned}
$$

The limit $u_{2} \rightarrow \infty$ yields the one-flavor result (62), i.e., $\Sigma_{1, N_{f}=2}^{d}\left(u_{1}, u_{2}=\infty, \theta\right)=\Sigma_{N_{f}=1}^{d}\left(u_{1}, \theta\right)$. This can readily be checked because only two terms of the integral are of leading order and the Bessel function $I_{\nu}$ can be approximated as in Eq. (80). In the limit $u_{1} \rightarrow \infty$ (at fixed $u_{2}$ ) one can show that $\Sigma_{1}^{d}$ is proportional to $1 / u_{1}$.

We emphasize that $\Sigma_{1}^{d}$ remains finite for large masses. The reason is the same as discussed in Sec. V, namely that the exponents of the $K_{\nu}$ and of the partition function cancel. Of course this should happen because the total chiral condensate, which can be obtained from the mass derivative (5) of the two-flavor partition function,

$$
\begin{aligned}
\frac{\Sigma_{1}(\vec{u}, \theta)}{\Sigma}= & \frac{d}{d u_{1}} \log Z(\vec{u}, \theta)=\frac{u_{1}+u_{2} \cos \theta}{\sqrt{u_{1}^{2}+u_{2}^{2}+2 u_{1} u_{2} \cos \theta}} \\
& \times \frac{I_{2}\left(\sqrt{u_{1}^{2}+u_{2}^{2}+2 u_{1} u_{2} \cos \theta}\right)}{I_{1}\left(\sqrt{u_{1}^{2}+u_{2}^{2}+2 u_{1} u_{2} \cos \theta}\right)},
\end{aligned}
$$

is finite. Although the expression for $\Sigma_{1}$ is quite complicated when derived via Eqs. (41) and (82), we checked numerically that it agrees with Eq. (83). (We did not succeed to give a direct analytical proof.) The behavior of $\Sigma_{1}$ is illustrated in Fig. 11 for several masses at the two angles $\theta=0$ and $\pi / 2$. It changes sign at $u_{1} / u_{2}=-\cos \theta$, which becomes the Dashen point $[19,29]$ for $\theta=0, \pi$ when taking the thermodynamic limit.
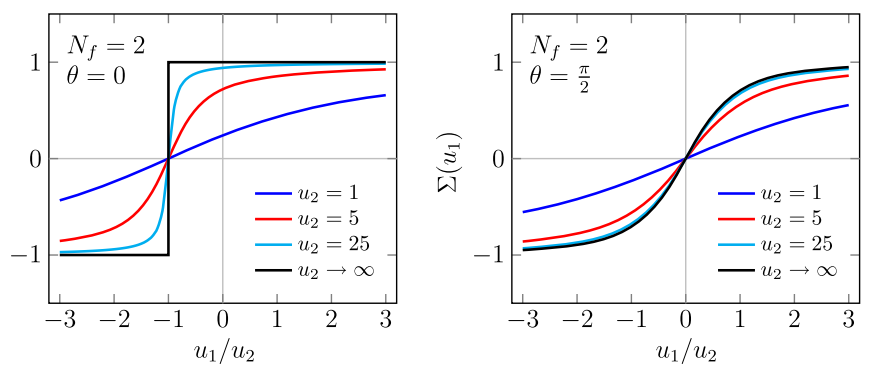

FIG. 11. Chiral condensate of the first quark in the two-flavor theory [see Eq. (83)] as a function of the first quark mass $u_{1}$ rescaled with respect to the fixed second quark mass $u_{2}$. The black curve is the thermodynamic limit. The jump in the left plot $(\theta=0)$ is the Dashen point [19,29], which corresponds to a first-order phase transition.
In Fig. 12 we show the decomposition of the chiral condensate for $\theta=0$ with one mass kept fixed at $u_{2}=20$. The sum of the quenched part and the zero-mode part, $\Sigma^{q}+\Sigma^{\mathrm{zm}}$, results in a chiral condensate with a discontinuity in the thermodynamic limit at $u_{1}=0$. Both parts become exponentially large in the volume when the product of the quark masses is negative, but their sum is finite. The dynamical part of the spectral density results in a chiral condensate with a discontinuity at $u_{1}=0$ that cancels the discontinuity of $\Sigma^{q}+\Sigma^{\mathrm{zm}}$ and creates a new discontinuity at $u_{1}=-u_{2}$.

\section{Thermodynamic limit}

Before we turn to the general setting, we first consider the cases $\theta=0$ with $u_{1} u_{2}>0$ and $\theta=\pi$ with $u_{1} u_{2}<0$, in which there is no sign problem. In the thermodynamic limit, the chiral condensate becomes

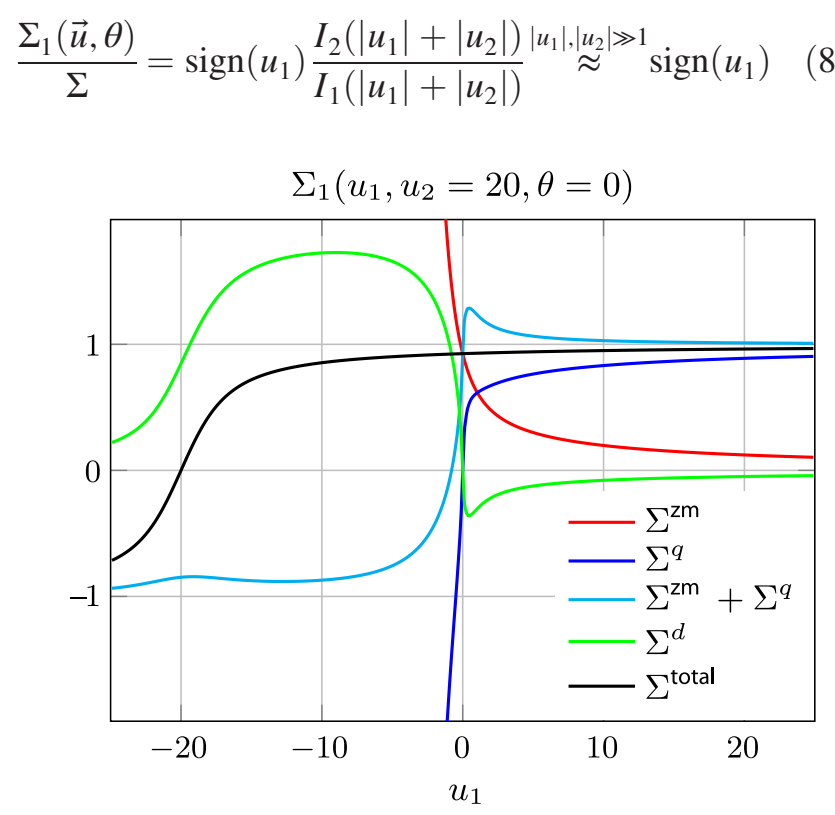

FIG. 12. Mass dependence of the various contributions to the chiral condensate for two-flavor $\mathrm{QCD}$ at $\theta=0$. The chiral condensate of the first quark is shown as a function of $u_{1}=m_{1} V \Sigma$, while the second mass is kept fixed at $u_{2}=20$. 


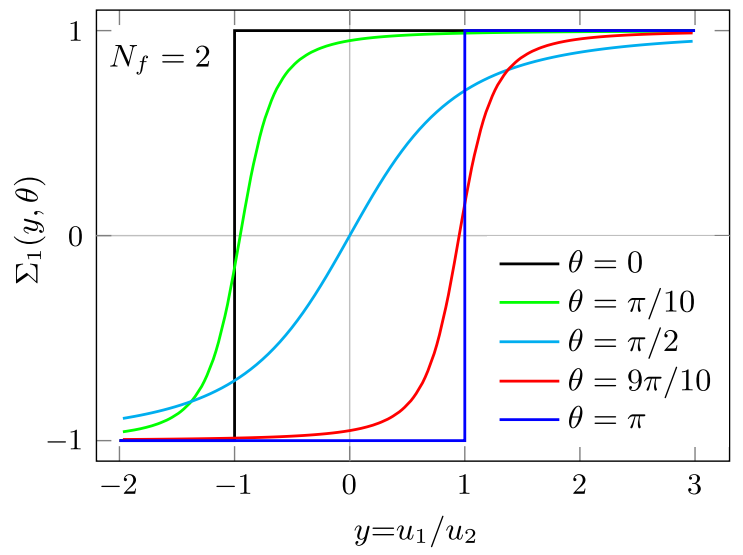

FIG. 13. Thermodynamic limit of the chiral condensate (87) as a function of the first quark mass $u_{1}$ for several $\theta$ angles and $u_{2}>0$. The chiral condensate depends only on the ratio $u_{1} / u_{2}$ in this particular limit. The Dashen point exists only for $\theta=0, \pi$ and shows up as a jump in the chiral condensate, reflecting the nature of a first-order phase transition.

because the leading asymptotic behavior of the Bessel functions does not depend on the index. Hence, its behavior is not different from the one-flavor case. The difference between the one- and two-flavor theory shows up in the zero-mode contribution, which is

$$
\frac{\Sigma_{1}^{\mathrm{zm}}(\vec{u}, \theta)}{\Sigma} \stackrel{\left|u_{1}\right|,\left|u_{2}\right| \gg>1}{\approx} \operatorname{sign}\left(u_{1}\right) \sqrt{\frac{2\left|u_{2}\right|}{\pi\left|u_{1}\right|\left(\left|u_{1}\right|+\left|u_{2}\right|\right)}} .
$$

To obtain this result for $\theta=0$ and $u_{1} u_{2}>0$ we have performed a saddle-point expansion of the integrand (81) about the point $\varphi=0$. Hence, we again have an algebraic dependence on the quark masses, which has a similar behavior as in the one-flavor case when both masses are equal, $\Sigma_{1}^{\mathrm{zm}}\left(\left|u_{1}\right|=\left|u_{2}\right|=u, 0\right) / \Sigma \approx 1 / \sqrt{\pi u}$; cf. Eq. (70).

The situation changes drastically when there is a sign problem, i.e., $\cos \theta \neq \operatorname{sign}\left(u_{1} u_{2}\right)$. In this case the zeromode contribution again exhibits exponential behavior,

$$
\begin{aligned}
\frac{\sum_{1}^{\mathrm{zm}}(\vec{u}, \theta)}{\Sigma} \approx & \frac{1}{\sqrt{8 \pi} \sin ^{2}\left[\frac{\theta}{2}+\left(1-\operatorname{sign}\left(u_{1} u_{2}\right)\right) \frac{\pi}{4}\right]} \frac{1}{u_{1}} \\
& \times \frac{\left(u_{1}^{2}+u_{2}^{2}+2 u_{1} u_{2} \cos \theta\right)^{3 / 2}}{\sqrt{\left|u_{1} u_{2}\right|}\left(\left|u_{1}\right|+\left|u_{2}\right|\right)} \\
& \times e^{\left|u_{1}\right|+\left|u_{2}\right|-\sqrt{u_{1}^{2}+u_{2}^{2}+2 u_{1} u_{2} \cos \theta}} .
\end{aligned}
$$

To obtain this result we performed a saddle-point approximation of Eq. (37) about the point $\varphi=\theta+$ $\left(1-\operatorname{sign}\left(u_{1} u_{2}\right)\right) \pi / 2$. Note that the derivation of (86) is only valid for both $\left|u_{1}\right| \gg 1$ and $\left|u_{2}\right| \gg 1$ and cannot be used in the chiral limit of any of the quark masses. For $\operatorname{sign}\left(u_{1} u_{2}\right)>0$ and $\theta \rightarrow \pi$, the exponential divergence in Eq. (86) is given by $\exp \left(2 \min \left\{\left|u_{1}\right|,\left|u_{2}\right|\right\}\right)$, which changes drastically at the point $\left|u_{1}\right|=\left|u_{2}\right|$ from $\exp \left(2\left|u_{1}\right|\right)$ to $\exp \left(2\left|u_{2}\right|\right)$.

The total chiral condensate still behaves algebraically in the masses,

$$
\begin{aligned}
& \frac{\Sigma_{1}(\vec{u}, \theta)}{\Sigma} \stackrel{\left|u_{1}\right|,\left|u_{2}\right| \gg 1}{\approx} \frac{u_{1}+u_{2} \cos \theta}{\sqrt{u_{1}^{2}+u_{2}^{2}+2 u_{1} u_{2} \cos \theta}} \\
& \quad=\operatorname{sign}\left(u_{2}\right) \frac{y+\cos \theta}{\sqrt{y^{2}+1+2 y \cos \theta}} \quad \text { with } y=\frac{u_{1}}{u_{2}} \\
& \quad= \begin{cases}\operatorname{sign}\left(u_{1}+u_{2}\right), & \theta=0, \\
\operatorname{sign}\left(u_{1}-u_{2}\right), & \theta=\pi .\end{cases}
\end{aligned}
$$

Apart from the factor $\operatorname{sign}\left(u_{2}\right)$, the thermodynamic limit of the chiral condensate is a function of $u_{1} / u_{2}$ only and is shown in Fig. 13 for several values of $\theta$. It changes sign at $u_{1} / u_{2}=-\cos \theta$. For $\theta \neq 0, \pi$ this transition is smooth, but for $\theta=0$ or $\theta=\pi$ (corresponding to the Dashen point $[19,29])$ it is discontinuous.

\section{CONCLUSIONS}

At nonzero $\theta$ angle, the discontinuity of the chiral condensate in general does not coincide with the support of the Dirac spectrum. In particular, for one-flavor QCD there is no discontinuity at zero quark mass, and for twoflavor QCD with quark masses $m_{1}$ and $m_{2}$, the chiral condensate of the first quark does not have a discontinuity at $m_{1}=0$ but rather at $m_{1}=-m_{2}$. We have analyzed this behavior in terms of the contribution from the zero modes, the contribution of the mass independent part of the Dirac spectrum (the "quenched" part), and the contribution of the remainder of the Dirac spectrum which is sensitive to the fermion determinant (at fixed topological charge).

At fixed $\theta$ angle, we have obtained a compact general formula for the contribution of the zero modes and of the quenched part of the Dirac spectrum to the chiral condensate. Both formulas are valid for any number of flavors and are given by an integral over a flavor independent kernel times the ratio of $N_{f}$ flavor partition functions. The formula for the zero modes is completely general, while the expression for the quenched part has been obtained in the microscopic domain of QCD but is also valid for any number of flavors. Both contributions diverge exponentially with the volume at nonzero $\theta$ angle, but the divergent contributions cancel identically when added, leaving a result that is finite in the thermodynamic limit. The deeper reason for the cancellation based on general ideas from spectral flow and topology is that when we have $|\nu|$ zero modes, the spectrum near zero is depleted by $|\nu|$ modes, half of them with positive eigenvalues and the other half with negative eigenvalues. This depletion gives 
a contribution to the chiral condensate with the opposite sign. For large $u=m V \Sigma$ it does not matter whether the modes are exactly at zero or are close to zero. The fermion determinant results in an additional eigenvalue repulsion from zero, which does not depend on $\nu$, and the contribution to the chiral condensate due to this modification of the Dirac spectrum is expected to remain finite in the thermodynamical limit.

For one and two flavors, we have also obtained exact analytical expressions for the dynamical part of the Dirac spectrum and the chiral condensate in the microscopic or $\varepsilon$ domain of QCD which confirm the above picture.

For $\theta \neq 0$ both the quenched and the dynamical contributions to the spectral density as well as their sum are strongly oscillating with an amplitude that diverges exponentially with the volume and a period on the order of $1 / \mathrm{V}$. From QCD at nonzero chemical potential we have learned that this behavior may cancel the discontinuity of the chiral condensate and shift it to a different point. The sum of the quenched part and the zero mode part retain the discontinuity at $m_{1}=0$, but the oscillations of the dynamical part cancels this discontinuity and move it to $m_{1}=-m_{2}$ for two flavors or to infinity for one flavor. The effect of the zero modes is to create a gap at zero but, in the quenched approximation, the position of the remaining eigenvalues does not depend very much on $\nu$. Since the condensate is obtained in the thermodynamical limit, it is not surprising that the quenched contributions to the chiral condensate have a discontinuity at $m_{1}=0$. We thus conclude that the determinant introduces correlations in the Dirac spectrum that cancel the discontinuity at $m_{1}=0$ and move it to $m_{1}=-m_{2}$. Currently we do not have a good understanding of the nature of these correlations, but hope to return to this issue in future work.

We have also seen that the correct computation of the chiral condensate at nonzero $\theta$ angle requires a subtle balance between zero and nonzero modes. Even the slightest incompatibilities will give results that are completely off. This will make lattice QCD simulations at nonzero $\theta$ angle a formidable, and perhaps impossible, task, and we have to rely on analytical work to make further progress.

\section{ACKNOWLEDGMENTS}

M. K. acknowledges support by the German Research Foundation (DFG) via CRC 1283: "Taming uncertainty and profiting from randomness and low regularity in analysis, stochastics and their applications." T. W. was supported by the DFG in the framework of SFB/TRR-55. J.V. was partially supported by U.S. DOE Grant No. DE-FAG88FR40388.

\section{APPENDIX A: RESUMMATION OF PRODUCTS OF BESSEL FUNCTIONS}

To compute the sums of Bessel functions needed in the main text we consider the sum

$$
\Xi_{a}^{(N)}(\theta, \vec{u})=\sum_{\nu=-\infty}^{\infty} e^{i \nu \theta} \prod_{j=1}^{N} I_{\nu+a_{j}}\left(u_{j}\right)
$$

where $I_{\nu}$ is the modified Bessel function of the first kind. The indices $a=\left(a_{1}, \ldots, a_{N}\right)$ are chosen to be integers while the masses $\vec{u}=\left(u_{1}, \ldots, u_{N}\right)$ can be arbitrary, even complex valued. Indeed we need imaginary masses $\vec{u}$ to generate the Bessel functions of the first kind via the relation $I_{\nu}(i z)=i^{\nu} J_{\nu}(z)$.

Using an integral representation of the Bessel function we can sum over $\nu$ employing the relation $\sum_{\nu=-\infty}^{\infty} e^{i \nu \varphi}=2 \pi \delta(\varphi)$. Note that the argument of the Dirac delta function has to be taken modulo $2 \pi$, which we omit. This sum yields

$\Xi_{a}^{(N)}(\theta, \vec{u})=2 \pi \prod_{j=1}^{N} \int_{-\pi}^{\pi} \frac{d \varphi_{j}}{2 \pi} e^{i a_{j} \varphi_{j}+u_{j} \cos \varphi_{j}} \delta\left(\sum_{k=1}^{N} \varphi_{k}+\theta\right)$.

The simplest case is $N=1$, for which

$$
\Xi_{a_{1}}^{(1)}\left(\theta, u_{1}\right)=e^{-i a_{1} \theta} e^{u_{1} \cos \theta} .
$$

For $a=0$ this corresponds to the one-flavor partition function

$$
Z\left(u_{1}, \theta\right)=\Xi_{0}^{(1)}\left(\theta, u_{1}\right)=e^{u_{1} \cos \theta} .
$$

For $N>1$, we define new variables $\vartheta_{1}=\varphi_{1}$ and $\vartheta_{j}=$ $\varphi_{j}+\vartheta_{j-1}$ for $j=2, \ldots, N$. This allows us to evaluate the delta function, and we end up with a chain of integrals,

$$
\begin{aligned}
\Xi_{a}^{(N)}(\theta, \vec{u})= & e^{-i a_{N} \theta} \prod_{j=1}^{N-1} \int_{-\pi}^{\pi} \frac{d \vartheta_{j}}{2 \pi} e^{i\left(a_{j}-a_{j+1}\right) \vartheta_{j}} \\
& \times e^{u_{1} \cos \vartheta_{1}+u_{N} \cos \left(\theta+\vartheta_{N-1}\right)+\sum_{k=2}^{N-1} u_{k} \cos \left(\vartheta_{k}-\vartheta_{k-1}\right)} .
\end{aligned}
$$

For general even $N$ we set $N=2 n$ with $n \in \mathbb{N}$ and integrate over $\vartheta_{1}, \vartheta_{3}, \ldots, \vartheta_{2 n-1}$. This again produces Bessel functions, 


$$
\begin{aligned}
\Xi_{a}^{(2 n)}(\theta, \vec{u})= & e^{-i a_{2 n} \theta} \prod_{j=1}^{n-1} \int_{-\pi}^{\pi} \frac{d \vartheta_{2 j}}{2 \pi} e^{i\left(a_{2 j}-a_{2 j+1}\right) \vartheta_{2 j}}\left(\frac{u_{1}+u_{2} e^{i \vartheta_{2}}}{u_{1}+u_{2} e^{-i \vartheta_{2}}}\right)^{\left(a_{1}-a_{2}\right) / 2} I_{a_{1}-a_{2}}\left(\sqrt{u_{1}^{2}+u_{2}^{2}+2 u_{1} u_{2} \cos \vartheta_{2}}\right) \\
& \times\left(\frac{u_{2 n-1} e^{i \vartheta_{2 n-2}}+u_{2 n} e^{-i \theta}}{u_{2 n-1} e^{-i \vartheta_{2 n-2}+u_{2 n} e^{i \theta}}}\right)^{\left(a_{2 n-1}-a_{2 n}\right) / 2} I_{a_{2 n-1}-a_{2 n}}\left(\sqrt{u_{2 n-1}^{2}+u_{2 n}^{2}+2 u_{2 n-1} u_{2 n} \cos \left(\vartheta_{2 n-2}+\theta\right)}\right) \\
& \times \prod_{k=1}^{n-2}\left(\frac{u_{2 k+1} e^{i \vartheta_{2 k}}+u_{2 k+2} e^{i \vartheta_{2 k+2}}}{u_{2 k+1} e^{-i \vartheta_{2 k}}+u_{2 k+2} e^{-i \vartheta_{2 k+2}}}\right)^{\left(a_{2 k+1}-a_{2 k+2}\right) / 2} I_{a_{2 k+1}-a_{2 k+2}}\left(\sqrt{u_{2 k+1}^{2}+u_{2 k+2}^{2}+2 u_{2 k+1} u_{2 k+2} \cos \left(\vartheta_{2 k}-\vartheta_{2 k+2}\right)}\right) .
\end{aligned}
$$

In the case of $N=2$, which is needed for the partition function of two flavors and can also be used for the quenched level density, we obtain

$$
\Xi_{a_{1}, a_{2}}^{(2)}\left(\theta, u_{1}, u_{2}\right)=e^{-i\left(a_{1}+a_{2}\right) \theta / 2}\left(\frac{u_{1} e^{i \theta}+u_{2}}{u_{2} e^{i \theta}+u_{1}}\right)^{\left(a_{1}-a_{2}\right) / 2} I_{a_{1}-a_{2}}\left(\sqrt{u_{1}^{2}+u_{2}^{2}+2 u_{1} u_{2} \cos \theta}\right) .
$$

Then the two-flavor partition function is

$$
Z\left(u_{1}, u_{2}, \theta\right)=2 \frac{u_{2} \Xi_{0,1}^{(2)}\left(\theta, u_{1}, u_{2}\right)-u_{1} \Xi_{1,0}^{(2)}\left(\theta, u_{1}, u_{2}\right)}{u_{2}^{2}-u_{1}^{2}}=\frac{2 I_{1}\left(\sqrt{u_{1}^{2}+u_{2}^{2}+2 u_{1} u_{2} \cos \theta}\right)}{\sqrt{u_{1}^{2}+u_{2}^{2}+2 u_{1} u_{2} \cos \theta}} .
$$

For $N=4$, which is needed for the level density with two flavors, the result is a single integral,

$$
\begin{aligned}
\Xi_{a}^{(4)}\left(\theta, u_{1}, u_{2}, u_{3}, u_{4}\right)= & e^{-i a_{4} \theta} \int_{-\pi}^{\pi} \frac{d \varphi}{2 \pi} e^{i\left(a_{2}-a_{3}\right) \varphi}\left(\frac{u_{1}+u_{2} e^{i \varphi}}{u_{1}+u_{2} e^{-i \varphi}}\right)^{\left(a_{1}-a_{2}\right) / 2}\left(\frac{u_{3} e^{i \varphi}+u_{4} e^{-i \theta}}{u_{3} e^{-i \varphi}+u_{4} e^{i \theta}}\right)^{\left(a_{3}-a_{4}\right) / 2} \\
& \times I_{a_{1}-a_{2}}\left(\sqrt{u_{1}^{2}+u_{2}^{2}+2 u_{1} u_{2} \cos \varphi}\right) I_{a_{3}-a_{4}}\left(\sqrt{u_{3}^{2}+u_{4}^{2}+2 u_{3} u_{4} \cos (\varphi+\theta)}\right) .
\end{aligned}
$$

For general odd $N$ we set $N=2 n+1$ with $n \in \mathbb{N}$ and again integrate over $\vartheta_{1}, \vartheta_{3}, \ldots, \vartheta_{2 n-1}$. This leads to a slightly different result,

$$
\begin{aligned}
\Xi_{a}^{(2 n+1)}(\theta, \vec{u})= & e^{-i a_{2 n+1} \theta} \\
& \times \prod_{j=1}^{n} \int_{-\pi}^{\pi} \frac{d \vartheta_{2 j}}{2 \pi} e^{i\left(a_{2 j}-a_{2 j+1}\right) \vartheta_{2 j}} e^{u_{2 n+1} \cos \left(\theta+\vartheta_{2 n}\right)}\left(\frac{u_{1}+u_{2} e^{i \vartheta_{2}}}{u_{1}+u_{2} e^{-i \vartheta_{2}}}\right)^{\left(a_{1}-a_{2}\right) / 2} I_{a_{1}-a_{2}}\left(\sqrt{u_{1}^{2}+u_{2}^{2}+2 u_{1} u_{2} \cos \vartheta_{2}}\right) \\
& \times \prod_{k=1}^{n-1}\left(\frac{u_{2 k+1} e^{i \vartheta_{2 k}}+u_{2 k+2} e^{i \vartheta_{2 k+2}}}{u_{2 k+1} e^{-i \vartheta_{2 k}}+u_{2 k+2} e^{-i \vartheta_{2 k+2}}}\right)^{\left(a_{2 k+1}-a_{2 k+2}\right) / 2} I_{a_{2 k+1}-a_{2 k+2}}\left(\sqrt{u_{2 k+1}^{2}+u_{2 k+2}^{2}+2 u_{2 k+1} u_{2 k+2} \cos \left(\vartheta_{2 k}-\vartheta_{2 k+2}\right)}\right) .
\end{aligned}
$$

For $N=3$, which is employed for the level density with one flavor, the result is a single integral,

$$
\Xi_{a}^{(3)}\left(\theta, u_{1}, u_{2}, u_{3}\right)=e^{-i a_{3} \theta} \int_{-\pi}^{\pi} \frac{d \varphi}{2 \pi} e^{i\left(a_{2}-a_{3}\right) \varphi} e^{u_{3} \cos (\theta+\varphi)}\left(\frac{u_{1}+u_{2} e^{i \varphi}}{u_{1}+u_{2} e^{-i \varphi}}\right)^{\left(a_{1}-a_{2}\right) / 2} I_{a_{1}-a_{2}}\left(\sqrt{u_{1}^{2}+u_{2}^{2}+2 u_{1} u_{2} \cos \varphi}\right) \text {. }
$$

The results above simplify further for the specific sums we are considering. For the contribution of the nonzero modes to the two-flavor level density we need $N=3$ and 4 with $u_{1}=u_{2}=i x$ and $\theta \rightarrow \theta-\pi$, i.e.,

$$
\begin{aligned}
\sum_{\nu=-\infty}^{\infty} e^{i \nu \theta} J_{\nu+a_{1}}(x) J_{\nu+a_{2}}(x) I_{\nu+a_{3}}(u) & =e^{-i \pi\left(a_{1}+a_{2}\right) / 2} \Xi_{a}^{(3)}(\theta-\pi, i x, i x, u) \\
& =(-1)^{a_{2}-a_{3}} e^{-i a_{3} \theta} \int_{-\pi}^{\pi} \frac{d \varphi}{2 \pi} e^{i\left(a_{1}+a_{2}-2 a_{3}\right) \varphi / 2} e^{-u \cos (\theta+\varphi)} J_{a_{1}-a_{2}}\left(2 x \cos \frac{\varphi}{2}\right)
\end{aligned}
$$


and

$$
\begin{aligned}
& \sum_{\nu=-\infty}^{\infty} e^{i \nu \theta} J_{\nu+a_{1}}(x) J_{\nu+a_{2}}(x) I_{\nu+a_{3}}\left(u_{1}\right) I_{\nu+a_{4}}\left(u_{2}\right)=e^{-i \pi\left(a_{1}+a_{2}\right) / 2} \Xi_{a}^{(4)}\left(\theta-\pi, i x, i x, u_{1}, u_{2}\right) \\
& =(-1)^{a_{2}+a_{4}} e^{-i\left(a_{3}+a_{4}\right) \theta / 2} \int_{-\pi}^{\pi} \frac{d \varphi}{2 \pi} e^{i\left(a_{1}+a_{2}-a_{3}-a_{4}\right) \varphi / 2}\left(\frac{u_{1} e^{i(\theta+\varphi)}-u_{2}}{u_{1}-u_{2} e^{i(\theta+\varphi)}}\right)^{\left(a_{3}-a_{4}\right) / 2} \\
& \times J_{a_{1}-a_{2}}\left(2 x \cos \frac{\varphi}{2}\right) I_{a_{3}-a_{4}}\left(\sqrt{u_{1}^{2}+u_{2}^{2}-2 u_{1} u_{2} \cos (\theta+\varphi)}\right) .
\end{aligned}
$$

\section{APPENDIX B: CALCULATION OF THE CONDENSATE $\Sigma_{1}^{d}$ FOR TWO FLAVORS}

The dynamical part of the level density is given by Eq. (76). We combine this result with Eq. (27). Thus we have to evaluate the integral

$$
\begin{aligned}
\Sigma_{1}^{d}(\vec{u}, \theta)= & \int_{0}^{\infty} d x \frac{2 u_{1}}{x^{2}+u_{1}^{2}} \rho^{d}(x, \vec{u}, \theta) \\
= & -\frac{2 u_{1}}{Z(\vec{u}, \theta)} \int_{0}^{\infty} d x \int_{-\pi}^{\pi} \frac{d \varphi}{2 \pi} \frac{x}{\left(x^{2}+u_{1}^{2}\right)^{2}\left(x^{2}+u_{2}^{2}\right)}\left\{x\left[2 u_{1} u_{2} \sin \frac{\theta-\varphi}{2}+\left(u_{2}^{2}+u_{1}^{2}\right) \sin \frac{\varphi}{2}\right] J_{1}\left(2 x \sin \frac{\varphi}{2}\right) Z(\vec{u}, \theta-\varphi)\right. \\
& \left.+2\left[x^{2} \cos \varphi+u_{1} u_{2} \cos (\theta-\varphi)\right] J_{0}\left(2 x \sin \frac{\varphi}{2}\right) I_{0}\left(\sqrt{u_{1}^{2}+u_{2}^{2}+2 u_{1} u_{2} \cos (\theta-\varphi)}\right)\right\} .
\end{aligned}
$$

As the first step we perform a partial fraction expansion of the ratios

$$
\begin{aligned}
\frac{1}{\left(x^{2}+u_{1}^{2}\right)^{2}\left(x^{2}+u_{2}^{2}\right)}= & \frac{1}{u_{2}^{2}-u_{1}^{2}}\left[\frac{1}{\left(x^{2}+u_{1}^{2}\right)^{2}}-\frac{1}{u_{2}^{2}-u_{1}^{2}} \frac{1}{x^{2}+u_{1}^{2}}+\frac{1}{u_{2}^{2}-u_{1}^{2}} \frac{1}{x^{2}+u_{2}^{2}}\right], \\
\frac{x^{2} \cos \varphi+u_{1} u_{2} \cos (\theta-\varphi)}{\left(x^{2}+u_{1}^{2}\right)^{2}\left(x^{2}+u_{2}^{2}\right)}= & \frac{u_{1} u_{2} \cos (\theta-\varphi)-u_{1}^{2} \cos \varphi}{\left(u_{2}^{2}-u_{1}^{2}\right)\left(x^{2}+u_{1}^{2}\right)^{2}}+\frac{u_{2}^{2} \cos \varphi-u_{1} u_{2} \cos (\theta-\varphi)}{\left(u_{2}^{2}-u_{1}^{2}\right)^{2}\left(x^{2}+u_{1}^{2}\right)} \\
& -\frac{u_{2}^{2} \cos \varphi-u_{1} u_{2} \cos (\theta-\varphi)}{\left(u_{2}^{2}-u_{1}^{2}\right)^{2}\left(x^{2}+u_{2}^{2}\right)}
\end{aligned}
$$

for the first and second terms in the integral, respectively. For the integral over the first term we need the integrals (C9) and (C10) while for the second term we employ (C11) and (C12). After some algebra we find Eq. (82).

\section{APPENDIX C: INTEGRALS OVER BESSEL FUNCTIONS}

At several places of our work we need recurrence relations and other identities of Bessel functions which can be found in [30,31]. We will briefly summarize those we need here. The ordinary and modified Bessel functions of the first kind satisfy the recurrence relations

$$
\begin{gathered}
x\left(J_{\nu+1}(x)+J_{\nu-1}(x)\right)=2 \nu J_{\nu}(x), \\
u\left(I_{\nu-1}(u)-I_{\nu+1}(u)\right)=2 \nu I_{\nu}(u), \\
\partial_{y} \frac{I_{\nu}(\sqrt{y})}{y^{\nu / 2}}=\frac{1}{2} \frac{I_{\nu+1}(\sqrt{y})}{y^{(\nu+1) / 2}} .
\end{gathered}
$$

The modified Bessel function of the second kind also satisfies two recursion relations [31],

$$
\begin{aligned}
x\left(K_{\nu+1}(x)-K_{\nu-1}(x)\right) & =2 \nu K_{\nu}(x), \\
K_{\nu+1}(x)+K_{\nu-1}(x) & =-2 \partial_{x} K_{\nu}(x) .
\end{aligned}
$$

Specifically, we have

$$
\begin{gathered}
\partial_{x} K_{0}(a x)=-a K_{1}(a x), \\
\partial_{x}\left[x K_{1}(a x)\right]=-a x K_{0}(a x) .
\end{gathered}
$$

Moreover we need the integral identities

$$
\int_{0}^{\infty} d x \frac{J_{1}(2 x t)}{x^{2}+u^{2}}=\frac{1}{2 t u^{2}}-\frac{\operatorname{sign}(t)}{|u|} K_{1}(2|t u|)
$$




$$
\begin{aligned}
& \int_{0}^{\infty} d x \frac{x^{2} J_{1}(2 x t)}{x^{2}+u^{2}}=\operatorname{sign}(t)|u| K_{1}(2|t u|), \\
& \int_{0}^{\infty} d x \frac{x^{2} J_{1}(2 x t)}{\left(x^{2}+u^{2}\right)^{2}}=t K_{0}(2|t u|), \\
& \int_{0}^{\infty} d x \frac{x J_{0}(2 x t)}{x^{2}+u^{2}}=K_{0}(2|t u|), \\
& \int_{0}^{\infty} d x \frac{x J_{0}(2 x t)}{\left(x^{2}+u^{2}\right)^{2}}=\frac{|t|}{|u|} K_{1}(2|t u|) .
\end{aligned}
$$

The first, second, and fourth integrals were also given in [11], where it was implicitly assumed that $t>0$.

\section{APPENDIX D: THERMODYNAMIC LIMIT OF $\Sigma^{d}$ FOR $N_{f}=1$}

In this Appendix we derive Eq. (72). We consider the RHS of (62), which we denote by $I$. For large argument the Bessel functions $K_{\nu}(x)$ can be approximated by $e^{-x} \sqrt{\pi / 2 x}$. Therefore, in a saddle-point approximation, the exponent to be analyzed is

$$
\begin{aligned}
f(\varphi) & =u \cos (\theta-\varphi)-u \cos \theta-2\left|u \sin \frac{\varphi}{2}\right| \\
& =-2\left|u \sin \frac{\varphi}{2}\right|\left[1-\operatorname{sign}\left(u \sin \frac{\varphi}{2}\right) \sin \left(\theta-\frac{\varphi}{2}\right)\right],
\end{aligned}
$$

which is always nonpositive and has a maximum of $f_{\max }(\varphi)=0$. A straightforward analysis shows that for $u \cos \theta>0$ the maximum is assumed only at $\varphi=0$, while for $u \cos \theta<0$ it is also assumed at $\bar{\varphi}=2 \theta-(2 k+1) \pi$, where $k \in \mathbb{Z}$ has to be chosen such that $\bar{\varphi} \in[-\pi, \pi]$. The latter is a true saddle point and dominates the integral. Expansion about $\bar{\varphi}$ yields to leading order in $|u|$,

$$
I \approx 2 \cos \theta \quad \text { (if } u \cos \theta<0)
$$

For $u \cos \theta>0$ we have to expand about $\varphi=0$. This is not a true saddle point since the derivative of $f(\varphi)$ is nonzero and discontinuous at this point. Furthermore, for $\varphi \rightarrow 0$ we cannot use the asymptotic expansion of $K_{\nu}(x)$.
Since the term involving $K_{0}$ comes with an additional factor of $\sin \frac{\varphi}{2}$ it is subleading and can be dropped. Hence, to leading order in $|u|$,

$$
\begin{aligned}
I & \approx-2 \int_{-\pi}^{\pi} \frac{d \varphi}{2 \pi} \frac{e^{u \cos (\theta-\varphi)}}{e^{u \cos \theta}}\left|u \sin \frac{\varphi}{2}\right| \cos \theta K_{1}\left(2\left|u \sin \frac{\varphi}{2}\right|\right) \\
& \approx-\frac{\cos \theta}{2 \pi|u|} \int_{-\infty}^{\infty} d t e^{t \sin \theta \operatorname{sign}(u)}|t| K_{1}(|t|) \\
& =-\frac{\cos \theta}{\pi|u|} \int_{0}^{\infty} d t \cosh (t \sin \theta) t K_{1}(t) \\
& =-\frac{\cos \theta}{2\left|u \cos ^{3} \theta\right|}=-\frac{1}{2 u \cos ^{2} \theta} \quad(\text { if } u \cos \theta>0),
\end{aligned}
$$

where the second line was obtained by transforming $\varphi=$ $t /|u|$ and expanding in $t$. The integral in the third line equals $\pi / 2\left(1-\sin ^{2} \theta\right)^{3 / 2}=\pi / 2|\cos \theta|^{3}$.

Finally we consider the case of $\cos \theta=0$. It is straightforward to show that the same result for $I$ is obtained for $\theta= \pm \pi / 2$ and that the result is odd in $u$. Hence we only consider $\theta=\pi / 2$ and $u>0$ in the following. For $\theta \rightarrow \pi / 2$ we have $\bar{\varphi} \rightarrow 0$; i.e., we again have to expand about $\varphi=0$. In this case we find for the exponent to leading order in $\varphi$

$$
f(\varphi) \approx \begin{cases}-\frac{1}{8} u \varphi^{3}, & \varphi>0 \\ 2 u \varphi, & \varphi<0\end{cases}
$$

The dominant contribution to the integral is thus obtained from the region $\varphi>0$. Since we are expanding for small $\varphi$, the term involving $K_{1}$ gives twice the result of the term involving $K_{0}$. Using the asymptotic expansion of $K_{\nu}$ we obtain to leading order in $|u|$

$$
\begin{aligned}
I & \approx-\frac{3 u}{\pi} \int_{0}^{\pi} d \varphi e^{-\frac{1}{8} u \varphi^{3}} \frac{\varphi^{2}}{4} \frac{\sqrt{\pi}}{\sqrt{2 u \varphi}} \\
& \approx-\frac{3}{\sqrt{\pi} u^{1 / 3}} \int_{0}^{\infty} d t e^{-t^{3}} t^{3 / 2} \\
& =-\frac{\Gamma(5 / 6)}{\sqrt{\pi} u^{1 / 3}} \quad(\text { if } \cos \theta=0 \text { and } u>0) .
\end{aligned}
$$

Observing that $I$ is odd in $u$ for $\cos \theta=0$ we obtain the last line of Eq. (72).
[1] G. 't Hooft, Phys. Rev. D 14, 3432 (1976); 18, 2199(E) (1978).

[2] A. A. Belavin, A. M. Polyakov, A. S. Schwartz, and Yu. S. Tyupkin, Phys. Lett. 59B, 85 (1975).
[3] D. J. Gross, R. D. Pisarski, and L. G. Yaffe, Rev. Mod. Phys. 53, 43 (1981).

[4] T. Schäfer and E. V. Shuryak, Rev. Mod. Phys. 70, 323 (1998). 
[5] H. Leutwyler and A. V. Smilga, Phys. Rev. D 46, 5607 (1992).

[6] V. Azcoiti, A. Galante, and V. Laliena, Prog. Theor. Phys. 109, 843 (2003).

[7] T. Kanazawa and A. Yamamoto, Phys. Rev. D 93, 016010 (2016).

[8] Y. Cai, T. Cohen, A. Goldbloom-Helzner, and B. McPeak, Phys. Rev. D 93, 114510 (2016).

[9] D. Gaiotto, Z. Komargodski, and N. Seiberg, J. High Energy Phys. 01 (2018) 110.

[10] Y. Tanizaki and Y. Kikuchi, J. High Energy Phys. 06 (2017) 102.

[11] J. J. M. Verbaarschot and T. Wettig, Phys. Rev. D 90, 116004 (2014).

[12] J. Verbaarschot and T. Wettig, Proc. Sci., LATTICE2014 (2014) 072.

[13] M. Kieburg, J. Verbaarschot, and T. Wettig, EPJ Web Conf. 175, 04004 (2018).

[14] E. V. Shuryak and J. J. M. Verbaarschot, Nucl. Phys. A560, 306 (1993).

[15] J. J. M. Verbaarschot, Phys. Rev. Lett. 72, 2531 (1994).

[16] T. Banks and A. Casher, Nucl. Phys. B169, 103 (1980).

[17] J. C. Osborn, K. Splittorff, and J. J. M. Verbaarschot, Phys. Rev. Lett. 94, 202001 (2005).

[18] T. Kanazawa, T. Wettig, and N. Yamamoto, J. High Energy Phys. 12 (2011) 007.
[19] R. F. Dashen, Phys. Rev. D 3, 1879 (1971).

[20] M. Creutz, Phys. Rev. Lett. 92, 201601 (2004).

[21] D. P. Horkel and S. R. Sharpe, Phys. Rev. D 90, 094508 (2014).

[22] J. J. M. Verbaarschot and I. Zahed, Phys. Rev. Lett. 70, 3852 (1993).

[23] P. H. Damgaard and S. M. Nishigaki, Nucl. Phys. B518, 495 (1998).

[24] T. Wilke, T. Guhr, and T. Wettig, Phys. Rev. D 57, 6486 (1998).

[25] C. Itzykson and J. B. Zuber, J. Math. Phys. 21, 411 (1980).

[26] L. Shifrin and J. J. M. Verbaarschot, Phys. Rev. D 73, 074008 (2006).

[27] A. D. Jackson, M. K. Sener, and J. J. M. Verbaarschot, Phys. Lett. B 387, 355 (1996).

[28] J. J. M. Verbaarschot, in Confinement, duality, and nonperturbative aspects of $Q C D$, Proceedings of the NATO Advanced Study Institute, Newton Institute Workshop, Cambridge, UK, 1997 (Springer, Heidelberg, 1998), pp. 343-378.

[29] M. Creutz, Ann. Phys. (Amsterdam) 339, 560 (2013).

[30] Wolfram Research, Inc., Mathematica version 9.0, 2012.

[31] I. S. Gradshteyn and I. M. Ryzhik, Table of Integrals, Series and Products 7th ed. (Academic Press, London, UK, 2007). 